This article has been accepted for publication in MNRAS (c) 2018 The Authors. Published by Oxford University Press on behalf of the Royal Astronomical Society. All rights reserved. 


\title{
Weak lensing light-cones in modified gravity simulations with and without massive neutrinos
}

\author{
Carlo Giocoli ${ }^{\oplus, 2,3 \star}$ Marco Baldi ${ }^{\oplus 1,2,3}$ and Lauro Moscardini ${ }^{1,2,3}$ \\ ${ }^{1}$ Dipartimento di Fisica e Astronomia, Alma Mater Studiorum Università di Bologna, via Gobetti 93/2, I-40129 Bologna, Italy \\ ${ }^{2}$ INAF - Osservatorio di Astrofisica e Scienza dello Spazio di Bologna, via Gobetti 93/3, I-40129 Bologna, Italy \\ ${ }^{3}$ INFN - Sezione di Bologna, viale Berti Pichat 6/2, I-40127 Bologna, Italy
}

Accepted 2018 September 5. Received 2018 September 4; in original form 2018 June 18

\begin{abstract}
We present a novel suite of cosmological $N$-body simulations called the DUSTGRAINpathfinder, implementing simultaneously the effects of an extension to general relativity in the form of $f(R)$ gravity and of a non-negligible fraction of massive neutrinos. We describe the generation of simulated weak lensing and cluster counts observables within a past lightcone extracted from these simulations. The simulations have been performed by means of a combination of the MG-GADGET code and a particle-based implementation of massive neutrinos, while the light-cones have been generated using the MAPSIM pipeline allowing us to compute weak lensing maps through a ray-tracing algorithm for different values of the source plane redshift. The mock observables extracted from our simulations will be employed for a series of papers focused on understanding and possibly breaking the well-known observational degeneracy between $f(R)$ gravity and massive neutrinos, i.e. the fact that some specific combinations of the characteristic parameters for these two phenomena (the $f_{R 0}$ scalar amplitude and the total neutrino mass $\Sigma m_{v}$ ) may result indistinguishable from the standard $\Lambda \mathrm{CDM}$ cosmology through several standard observational probes. In particular, in this work we show how a tomographic approach to weak lensing statistics could allow - especially for the next generation of wide-field surveys - to disentangle some of the models that appear statistically indistinguishable through standard single-redshift weak lensing probe.
\end{abstract}

Key words: gravitational lensing: weak-methods: numerical-galaxies: haloes-dark energy-dark matter-cosmology: theory.

\section{INTRODUCTION}

General relativity predicts that light rays are bent by the matter density distribution analogously to what happens in optics when light rays travel through media characterized by different diffraction indices (Einstein 1918; Landau \& Lifshitz 1971).

Light emitted by a distant galaxy, travelling for different gigaparsecs, tends to be deflected multiple times depending on the intervening matter density distribution along the line of sight. This effect - termed gravitational lensing - can be observed as a modification of the intrinsic shape of the background galaxy caused by the different paths followed by the various light rays emitted by its various components (Bartelmann \& Schneider 2001; Bartelmann 2010).

When rays pass close to a very massive and compact object - the centre of a massive galaxy or a galaxy cluster - they are strongly deflected and sometimes even follow multiple paths. The resulting galaxy image appears highly distorted into a gravitational arc or multiply imaged in different positions, depending on the optical lensing configuration: relative position between the observer, the lens, and the source (Broadhurst et al. 2005b; Coe et al. 2013; Meneghetti et al. 2013). This regime is termed strong gravitational lensing. When light bundles travel sufficiently far from any compact mass distribution they tend to be only slightly deflected (Bartelmann 2010). In this case the intrinsic shape of the source is only marginally modified: we are in the regime of weak gravitational lensing. Considering that by definition the weak lensing effect is tiny, it is necessary to average on a large number of background sources in order to quantify it and to indirectly perform a reasonable reconstruction of the intervening matter density distribution along the line of sight (for a review see Kilbinger 2015). In this direction many ongoing and planned future surveys are aimed to collect the largest possible number of galaxy images to measure their ellipticities and statistically infer their tiny distortions so as to obtain a solid estimate of the weak lensing effect. The future ESA-mission Euclid $^{1}$ (Laureijs et al. 2011) is expected to increase the number of 
weak lensing sources by more than a factor of four/five and cover a much larger area with respect to ground-based wide-field surveys like CFHTLenS (Benjamin et al. 2013; Erben et al. 2013) and KiDS (Hildebrandt et al. 2017) - and to extend their distribution to significantly higher redshifts (Kitching et al. 2016). All this will translate in an unprecedented capability of performing systematic analyses of cosmic weak gravitational lensing, on large field of view, and accurately trace the growth of non-linear structures down to small scales. The large number of background galaxies will allow also to perform weak lensing tomographic analyses, on a large number of redshift bins, tracing the growth of structures up to high redshifts.

Gravitational lensing probes the dynamics and the kinematics of the Universe and its large-scale structures, and for this reason it represents an unbiased tool to probe gravity on cosmological distances. The need to test General Relativity (hereafter GR) beyond the small scales of the Solar System has recently become an urgent task for the scientific community, mainly with the aim of finding possible alternative scenarios to the simple cosmological constant as a source of the late-time accelerated expansion of the Universe (Hu \& Sawicki 2007; Bertschinger \& Zukin 2008; Schmidt 2008; Novikov 2016). In this context, in order to be viable a Modified Gravity (MG, hereafter) model must produce an expansion history that does not deviate too much from that of the standard $\Lambda \mathrm{CDM}$ cosmology, while allowing for deviations in the dynamical evolution of density perturbations through gravitational instability. Such deviations need nonetheless to vanish (and GR must be recovered) in our local neighbourhood in order to fulfill the tight bounds on GR derived within the Solar System (Bertotti, Iess \& Tortora 2003; Will 2005). The mechanism to achieve such recovery is generally known as Screening (see e.g. Deffayet et al. 2002; Khoury \& Weltman 2004; Hinterbichler \& Khoury 2010; Brax \& Valageas 2014) and acts at non-linear scales as a suppression of the relative size of the MG fifth-force when the gravitational potential or its derivative become large. In this context, numerical simulations of MG models (see e.g. Oyaizu, Lima \& Hu 2008; Schmidt et al. 2009; Li et al. 2012; Puchwein, Baldi \& Springel 2013; Llinares, Mota \& Winther 2014; Arnold et al. 2018) represent the best phenomenological laboratories to study 'where to look at' to find deviations from GR, as they properly account for the full non-linear evolution of structures that is responsible for the onset of the Screening effect. In particular, statistical analyses of gravitational lensing within simulated light-cones are able to probe, as a function of the angular scale, the integrated effect of the fifth force caused by a given modification of gravity from linear down to non-linear scales (Harnois-Déraps et al. 2015). Recently, Barreira et al. (2017) have constructed past light-cones from MG simulations finding that non-standard physics can leave signatures both in the cosmic shear power spectrum and in the projected galaxy density profile. Other interesting results on past light-cones simulations of $f(R)$ models have also been obtained by Higuchi \& Shirasaki (2016), Shirasaki et al. (2017), and Li \& Shirasaki (2018).

The recent tensions between high- and low-redshift probes advocated by the Planck CMB (Planck Collaboration I 2011, 2014, 2016), Planck cluster counts (Planck Collaboration XI 2013; Planck Collaboration I 2014; Planck Collaboration XXIV 2016a), and weak lensing surveys like CFHTLens (Fu et al. 2008; Heymans et al. 2013; Kilbinger et al. 2013; Kitching et al. 2014) and KiDS (Hildebrandt et al. 2017) have increased the interest about non-standard models as possible solution for attenuating those tensions. Many works (Lesgourgues \& Pastor 2006; Costanzi et al. 2014; Poulin et al. 2018) have suggested that the presence of massive neutrinos can help reducing these tensions. Solar neutrino oscillations (Cleveland et al. 1998) have revealed the presence of massive neutrinos families, suggesting that a (yet undetermined) fraction of the total matter density of the Universe must be associated with the cosmic neutrino background.

In recent years, significant progress has been made in including the effects of massive neutrinos into cosmological $N$-body codes employed to study structure formation processes from the linear to the highly non-linear regime in the context of standard $\Lambda \mathrm{CDM}$ cosmologies (Viel, Haehnelt \& Springel 2010; Wagner, Verde \& Jimenez 2012; Villaescusa-Navarro et al. 2018; Zennaro et al. 2017). In this work we extend such analysis to MG cosmologies for a particular choice of an MG scenario, namely the $f(R)$ gravity (Buchdahl 1970; Starobinsky 1980; Hu \& Sawicki 2007; Sotiriou \& Faraoni 2010) by developing a series of $N$-body simulations performed with an extended version of the MG code MG-GADGET (Puchwein et al. 2013) that includes at the same time the particle-based implementation of massive neutrinos developed by Viel et al. (2010). Some early numerical investigations performed with such extended code (see Baldi et al. 2014) have highlighted a strong observational degeneracy between the effects of $f(R)$ gravity and those of massive neutrinos on structure formation processes over a wide range of scales and redshifts, covering both the linear and nonlinear regimes. In this context, a degeneracy means the property of two cosmological models characterized by fundamentally different laws of physics or energy content being indistinguishable from each other within observational errors. In particular, Baldi et al. (2014) showed that a proper combination of $f(R)$ gravity parameters and of the total neutrino mass $\Sigma m_{v}$ may result in basic observables like the non-linear matter power spectrum, the halo abundance, and the halo bias to be statistically consistent with $\Lambda$ CDM. Testing the use of weak lensing tomography as a probe to disentangle degenerate cosmological scenarios will be one of the main goals of this work. Our simulations - called the DUSTGRAIN-pathfinder - will allow us to investigate the joint effects of $f(R)$ gravity, and massive neutrinos in the non-linear regime of structure formation and systematically study particular non-standard degenerate cosmological models through weak lensing and halo counts statistics with the aim of shedding some light onto which statistics may help us disentangle such degenerate models from the standard $\Lambda \mathrm{CDM}$ one. We based our investigation on the analysis of past light-cones extracted from the simulations within which we computed the galaxy cluster distribution and the projected cosmic shear maps. The simulations presented here will be the base of a series of papers focusing on various different observational probes (Hagstotz et al. 2018; Peel et al. 2018)

Our paper is organized as follows. In Section 2 we describe our numerical simulations, their post-processing procedures, and the past light-cones constructed in the various runs. Section 3 is devoted to discuss our results on the halo mass functions in the past light-cone (3.1) and on the properties of the convergence maps (3.2): power spectra, tomographic analyses, and one point statistics. In Section 4 we summarize our results and draw our conclusions.

\section{NUMERICAL SIMULATIONS}

\subsection{The DUSTGRAIN-pathfinder simulations}

For the analysis discussed in this work we make use of the projected matter distribution and halo catalogues extracted from a suite of cosmological dark matter-only simulations called the DUSTGRAINpathfinder runs. The DUSTGRAIN (Dark Universe Simulations to Test GRAvity In the presence of Neutrinos) project is an ongoing 
enterprise aimed at producing large and detailed mock observations of galaxy clustering, weak lensing, CMB lensing, and redshift-space distortions in the context of cosmological models characterized by a modification of the laws of gravity from their standard GR form and by a non-negligible fraction of the cosmic matter density being made of standard massive neutrinos. For the former we consider an $f(R)$ gravity theory defined by the action (Buchdahl 1970):

$S=\int \mathrm{d}^{4} x \sqrt{-g}\left(\frac{R+f(R)}{16 \pi G}+\mathcal{L}_{m}\right)$,

where we assume for the $f(R)$ function the widely considered form (Hu \& Sawicki 2007):

$f(R)=-m^{2} \frac{c_{1}\left(\frac{R}{m^{2}}\right)^{n}}{c_{2}\left(\frac{R}{m^{2}}\right)^{n}+1}$,

with $R$ being the Ricci scalar curvature, $m^{2} \equiv H_{0}^{2} \Omega_{\mathrm{M}}$ being a mass scale, while $c_{1}, c_{2}$, and $n$ are non-negative constant free parameters of the model. Here we focus on the specific case for which the background expansion history is fixed to the standard $\Lambda \mathrm{CDM}$ one by choosing $c_{1} / c_{2}=6 \Omega_{\Lambda} / \Omega_{\mathrm{M}}-$ where $\Omega_{\Lambda}$ and $\Omega_{\mathrm{M}}$ represent the vacuum and matter energy density, respectively, under the condition $c_{2}\left(R / m^{2}\right)^{n} \gg 1$, so that the scalar field $f_{R}$ takes the approximate form:

$f_{R} \approx-n \frac{c_{1}}{c_{2}^{2}}\left(\frac{m^{2}}{R}\right)^{n+1}$.

We restrict our analysis to the case $n=1$ so that the model is left with only one free parameter, which can be expressed as

$f_{R 0} \equiv-\frac{1}{c_{2}} \frac{6 \Omega_{\Lambda}}{\Omega_{M}}\left(\frac{m^{2}}{R_{0}}\right)^{2}$.

As it is now generally accepted (He 2013; Motohashi, Starobinsky \& Yokoyama 2013; Baldi et al. 2014; Wright, Winther \& Koyama 2017), MG theories such as e.g. $f(R)$ gravity in the $\mathrm{Hu}$ $\&$ Sawicki form are strongly degenerate in a wide range of their observable footprints with the effects of massive neutrinos on structure formation, posing serious challenges to present and future large galaxy surveys in devising robust and reliable methods to disentangle the two phenomena. In particular, standard statistics such as the matter autopower spectrum, the lensing convergence power spectrum, and the halo mass function may be hardly distinguishable from their standard $\Lambda \mathrm{CDM}$ expectation for some specific combinations of the $f(R)$ gravity parameter $f_{R 0}$ and of the total neutrino mass $m_{v} \equiv \Sigma m_{v, i}$ (see Baldi et al. 2014; Peel et al. 2018) which define a 'maximum degeneracy' relation between the two models.

As the degeneracy is mostly driven by the non-linear behaviour of both the MG and the massive neutrinos effects on structure formation, linear tools are not suitable to properly explore the combined parameter space and identify the shape of such 'maximum degeneracy' relation. Therefore, in order to set the specific parameter combinations for the full DUSTGRAIN runs it was necessary to run a suite of smaller-scale and lower-resolution $N$-body simulations - called the pathfinder runs - to (at least coarsely) sample the $\left\{f(R), m_{v}\right\}$ parameter space. While the full-scale DUSTGRAIN simulations are still running at the time of writing this paper, the pathfinder runs already provide a wealth of valuable simulated data and of novel information content to deserve a detailed analysis. We start such investigations with this work focusing on weak lensing tomography on the past light-cone and its correlations with the distribution of massive clusters and with two companion papers focusing on higher-order statistics of weak lensing and on the modelling of the halo mass function in these cosmologies (Hagstotz et al.
2018; Peel et al. 2018, respectively). These will be followed soon by a series of other papers targeted at other types of observables.

In the Newtonian gauge, we can write the perturbed metric as

$\mathrm{d} s^{2}=(1+2 \Psi) \mathrm{d} t^{2}-a^{2}(t)(1-2 \Phi) \mathrm{d} x_{i} \mathrm{~d} x_{j}$,

where $\Phi$ and $\Psi$ are the Einstein-frame metric gravitational potentials. In standard GR model, where the accelerated expansion of the Universe is driven by a cosmological constant we can read the two potentials as

$\Phi=\Psi=\Psi_{\mathrm{N}}$,

where the Newton potential $\Psi_{\mathrm{N}}$ can be written in terms of the Poisson equation as

$\frac{\nabla^{2} \Psi_{\mathrm{N}}}{a^{2}}=4 \pi G \delta \rho=\frac{3 \Omega_{\mathrm{m}} H_{0}^{2}}{2 a^{3}} \delta$.

The symbols $\Omega_{\mathrm{M}}$ and $H_{0}$ represent the total matter density parameter and the Hubble constant at the present time, respectively, and $\delta \equiv$ $\delta \rho / \bar{\rho}$ is the matter density contrast.

In the $f(R)$ MG models the variation of the Einstein equations leads to the two modified Poisson equations for the metric potentials:

$\frac{\nabla^{2} \Phi}{a^{2}}=-\frac{c^{2} \nabla^{2}}{2 a^{2}} \delta f_{\mathrm{R}}+4 \pi G \delta \rho$

$\frac{\nabla^{2} \Psi}{a^{2}}=\frac{c^{2} \nabla^{2}}{2 a^{2}} \delta f_{\mathrm{R}}+4 \pi G \delta \rho$,

which can be written in terms of the Newton potential as

$\Phi=\Psi_{\mathrm{N}}-\frac{c^{2}}{2} \delta f_{\mathrm{R}}, \quad \Psi=\Psi_{\mathrm{N}}+\frac{c^{2}}{2} \delta f_{\mathrm{R}}$.

At this point we can introduce the lensing potential $\Phi_{1}$ as

$\Phi_{1}=\frac{\Phi+\Psi}{2}$

from which we can notice that the two extra terms in MG models cancel out: the lensing potential in $f(R)$ gravity remains unchanged from its standard GR form. Therefore, we can define the convergence $\kappa$ in the usual way as

$\kappa(\theta)=\int_{0}^{\infty} \mathrm{d} w \frac{w}{c^{2}} g(w) \nabla^{2} \Phi_{1}(w, w \theta)$,

where $w$ represents the comoving radial distance and $g(w)$ the survey weight function.

Coming to a technical description of the DUSTGRAINpathfinder runs, these are cosmological collisionless simulations following the evolution of an ensemble of $768^{3}$ dark matter particles of mass $m_{\mathrm{CDM}}=8.1 \times 10^{10} \mathrm{M}_{\odot} h^{-1}$ (for the case of $m_{v}=0$ ) and of as many neutrino particles (for the case of $m_{v}>0$ ) within a periodic cosmological box of $750 \mathrm{Mpc} h^{-1}$ per side, under the effect of a gravitational interaction dictated by equation (1). Standard cosmological parameters are set to be consistent with the Planck 2015 constraints (Planck Collaboration XIII 2016b), namely $\Omega_{\mathrm{M}}=$ $\Omega_{\mathrm{CDM}}+\Omega_{\mathrm{b}}+\Omega_{v}=0.31345, \Omega_{\mathrm{b}}=0.0481, \Omega_{\Lambda}=0.68655, H_{0}$ $=67.31 \mathrm{~km} \mathrm{~s}^{-1} \mathrm{Mpc}^{-1}, \mathcal{A}_{\mathrm{s}}=2.199 \times 10^{-9}, n_{\mathrm{s}}=0.9658$ which give at $z=0$ a root-mean-square of the linear density fluctuation smoothed on a scale of $8 \mathrm{Mpc} h^{-1}$ equal to $\sigma_{8}=0.847$.

For these simulations we employed the MG-Gadget code (see Puchwein et al. 2013), a modified version of the GADGET code (Springel 2005) that implements the extra force and the Chameleon screening mechanism (see Khoury \& Weltman 2004) that characterize $f(R)$ gravity by solving the non-linear Poisson-like equation 
Table 1. The subset of the DUSTGRAIN-pathfinder simulations considered in this work with their specific parameters, $f_{R 0}$ represents the MG parameter, $m_{\nu}$ and $m_{v}^{p}$ the neutrino mass in electron volt and in $m_{\odot} h^{-1}$ as implemented in the simulation, $m_{\mathrm{CDM}}^{p}$ CDM particle mass, and $\Omega_{\mathrm{CDM}}$ and $\Omega_{v}$ the CDM and neutrino density parameters, respectively. In the last column of the table we display the $\sigma_{8}$ parameter at $z=0$ for the different cosmological models computed from linear theory.

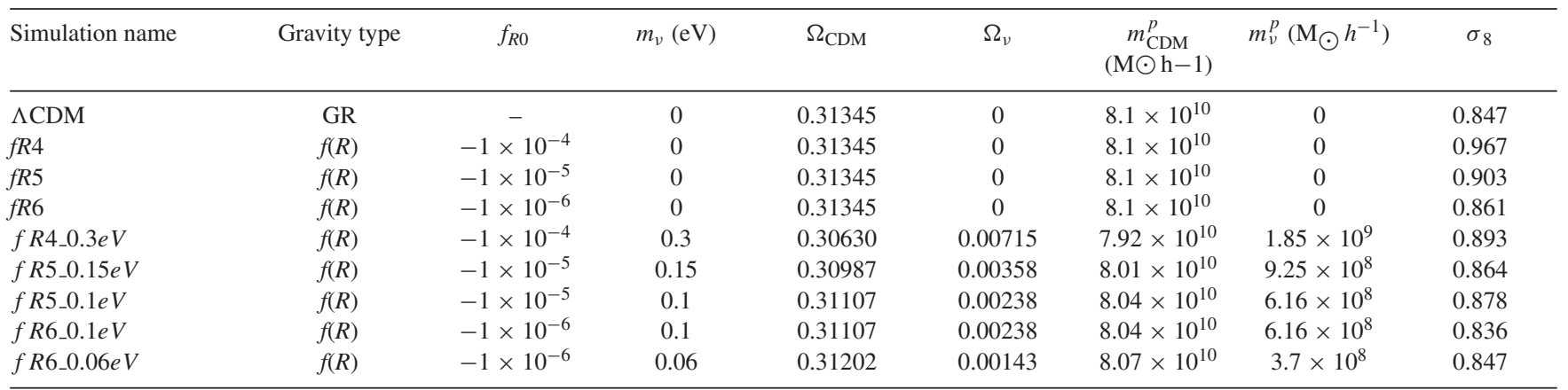

for the $f_{R}$ scalar degree of freedom

$\nabla^{2} f_{R}=\frac{1}{3}(\delta R-8 \pi G \delta \rho)$,

through a Newton-Gauss-Seidel iterative scheme on the native gravitational Tree of GADGET which is exploited as an adaptive mesh (see Puchwein et al. 2013, for more details about MGGADGET). MG-Gadget code has been extensively tested (see e.g. the MG code comparison project described in Winther et al. 2015) and employed in the recent past for a wide variety of applications ranging from large-scale collisionless cosmological simulations (Arnold et al. 2018; Baldi \& Villaescusa-Navarro 2018) to hydrodynamical simulations (Arnold, Puchwein \& Springel 2014; Arnold, Puchwein \& Springel 2015; Roncarelli, Baldi \& Villaescusa-Navarro 2018), to zoomed simulations of Milky Waysized objects (Arnold, Springel \& Puchwein 2016; Naik et al. 2018). In this work, following the approach already adopted in Baldi et al. (2014) we have combined the MG-Gadget solver with the particlebased implementation of massive neutrinos developed by Viel et al. (2010) in order to include massive neutrinos in our simulations as an additional family of particles with its specific initial transfer function and velocity distribution. Therefore, both cold dark matter $(\mathrm{CDM})$ and neutrino particles contribute to the density source term for the scalar perturbations evolution on the right-hand side of equation (13).

Initial conditions have been generated following the approach of e.g. Zennaro et al. (2017), Villaescusa-Navarro et al. (2018) which amounts to generating two fully correlated random realizations of the linear matter power spectrum for standard CDM particles and massive neutrinos based on their individual transfer functions computed by the linear Boltzmann code CAMB (Lewis, Challinor \& Lasenby 2000) at the starting redshift of the simulation $z_{\mathrm{i}}=99$, and by computing the scale-dependent growth rate $D_{+}\left(z_{\mathrm{i}}, k\right)$ for the neutrino component in order to correctly compute neutrino gravitational velocities. Thermal neutrino velocities are then added on top of the latter by random sampling of the neutrino momentum distribution at the starting redshift $z_{\mathrm{i}}$ for the specific neutrino mass under consideration.

The DUSTGRAIN-pathfinder simulations spanned the parameter range $-1 \times 10^{-4} \leq f_{R 0} \leq-1 \times 10^{-6}$ for the scalar amplitude and $0 \mathrm{eV} \leq m_{v} \leq 0.3 \mathrm{eV}$ for the neutrino mass with 20 different parameter combinations. In this work, we restrict the analysis to a subset of these simulations including three pure $f(R)$ runs (i.e. for $m_{v}$ $=0 \mathrm{eV})$ and those of their massive neutrino counterparts that result in a significant observational degeneracy amounting to one or two values of $m_{v}$ for each $f_{R 0}$ value, plus a standard $\Lambda$ CDM simulation (i.e. GR and massless neutrinos) as a reference model, for a total of nine simulations that are summarized in Table 1 . In the last column we show the $\sigma_{8}$ parameter for the different cosmological models. In particular, for the $f(R)$ models we have computed $\sigma_{8}$ from linear theory using MG-CAMB (Zhao et al. 2009; Hojjati, Pogosian \& Zhao 2011), while for the combined models of massive neutrinos plus $f(R)$ we joined together the predictions of CAMB for massive neutrinos with those of MG-CAMB. For all simulations we stored 34 full snapshots for a set of redshifts that allows to construct without any gap the lensing light-cones up to $z_{\mathrm{s}}=4$ that are described below.

\subsection{The halo catalogues}

For all simulations we have identified collapsed CDM structures in each comoving snapshot by means of a Friends-of-Friends algorithm (FoF, Davis et al. 1985) run on the $\mathrm{CDM}^{2}$ particles with linking length $\lambda=0.16 \times d$, where $d$ is the mean interparticle separation, retaining only structures with more than $32 \mathrm{CDM}$ particles. On top of such FoF catalogues we have run the SUBFIND algorithm (Springel et al. 2001) to identify gravitationally bound structures and to associate standard quantities such as the virial mass $M_{200}$ and the virial radius $R_{200}$ to the main substructure of each FoF group. The latter quantities are computed in the usual way by growing spheres of radius $R$ around the most-bound particle of each main substructure enclosing a total mass $M$ until the relation

$\frac{4}{3} \pi R_{200}^{3} \times 200 \times \rho_{\text {crit }}=M_{200}$

is fulfilled for $R=R_{200}$ and $M=M_{200}$, where $\rho_{\text {crit }} \equiv 3 H^{2} / 8 \pi G$ is the critical density of the universe.

\subsection{Past light-cones}

Several possible approaches can be followed to extract lensing light-cones from large cosmological $N$-body simulations. Recent efforts in the context of MG simulations have employed both postprocessing reconstructions based on the slicing of a set of comoving particle snapshots (as e.g. in Shirasaki et al. 2017) as well as more efficient on-the-fly algorithms capable of storing only the projected

${ }^{2}$ For the simulations containing also massive neutrinos we decided to follow the approach of not linking them to the collapsed haloes. 

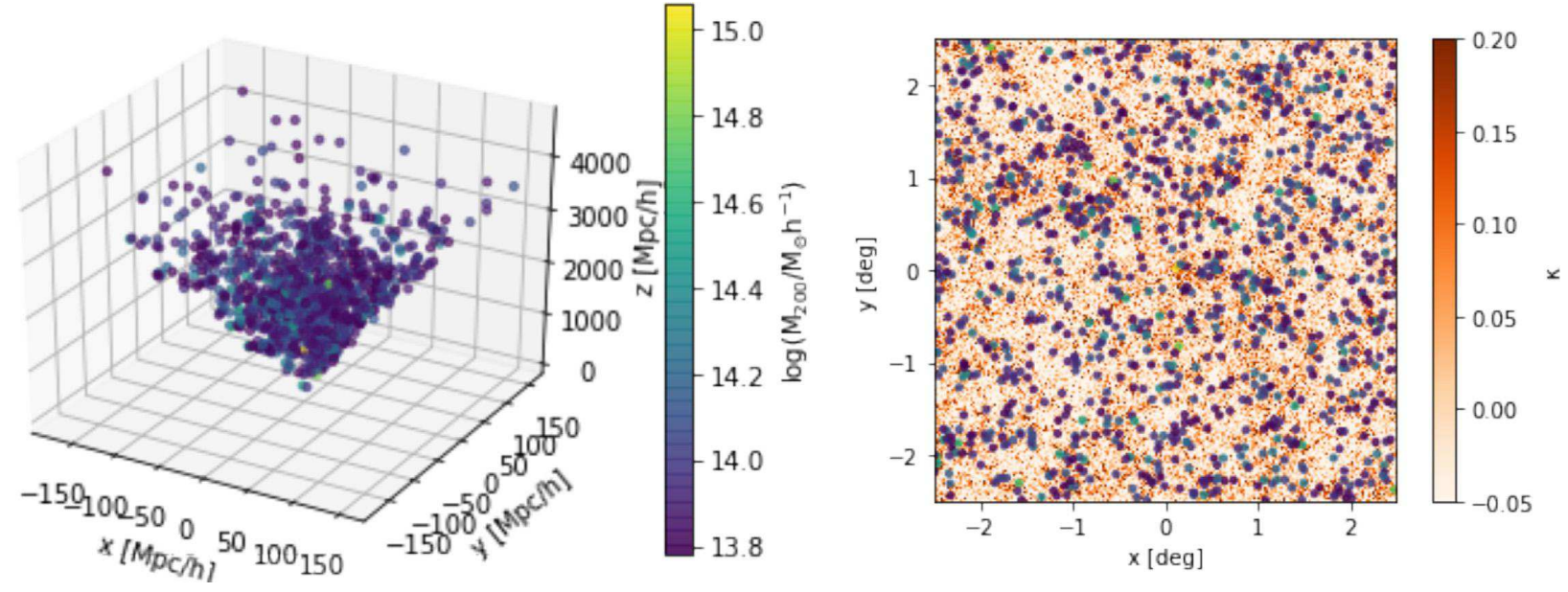

Figure 1. Schematic representation of the past light-cone using our MAPSIM routine. Left-hand panel shows the 3D distribution of haloes within the light-cone with $5 \times 5 \mathrm{deg}^{2}$ aperture up to redshift $z=4$. We display all haloes with mass larger than $M_{200} \geq 6 \times 10^{13} M_{\odot} h^{-1}$ colour coded according to their mass. Right-hand panel shows the convergence map for $z_{\mathrm{s}}=4$ - which represents the base of our past light pyramid - on which we also display the projected distribution of the haloes present inside the field of view. The scale colour of the underlining map refers to the convergence value corresponding to each pixel.

matter density on a given field of view without resorting on the flat-sky approximation (see e.g. Barreira et al. 2016; Arnold et al. 2018). While for the full-scale DUSTGRAIN simulations the latter approach will be also adopted - following in particular the same implementation of Arnold et al. (2018) - for the present analysis of the pathfinder runs we stick to the former procedure, and for each $\mathrm{N}$-body simulation we build the past light-cones using the MAPSIM routine (Giocoli et al. 2014). The particles from various snapshots are distributed onto different lens planes according to their comoving distances with respect to the observer and to whether they lie within a defined aperture of the field of view. We use the particles stored in 21 different snapshots to construct continuous past light-cones from $z=0$ to $z=4$, with a square sky coverage of 5 deg by side. Considering the good time resolution with which the snapshots have been stored, we are able to build 27 lens planes for the projected matter density distribution. In MAPSIM the observer is placed at the vertex of a pyramid whose square base is at the comoving distance corresponding to $z=4$. For each cosmological model we construct 256 different light-cone realizations by randomizing the various comoving cosmological boxes through combinations of the following procedures: (i) changing sign of the Cartesian coordinates; (ii) redefining the position of the observer, and (iii) modifying the order of the axes in the coordinate system. By construction, these variations preserve the clustering properties of the particle distribution at a given simulation snapshot (Roncarelli et al. 2007). The recent improvements of MAPSIM (Giocoli et al. 2017; Castro et al. 2018) give us also the possibility to store the corresponding halo and subhalo catalogues associated with a specific particle randomization of the past light-cone.

The MAPSIM pipeline allows us the construct lensing planes from different simulation snapshots, saving for each plane $l$, on each pixel, with coordinate indices $(i, j)$, the particle surface mass density $\Sigma$ :

$\Sigma_{l}(i, j)=\frac{\sum_{k} m_{k}}{A_{l}}$

where $A_{l}$ represents the comoving pixel area of the $l$-lens plane and $\sum_{k} m_{k}$ the sum on all particle masses associated with the pixel. Being gravitational lensing sensitive to the projected matter density distribution along the line of sight, onto each lens plane we project all particles between two defined comoving distances from the ob- server; in the simulations with massive neutrinos we consistently account also for this component. As done by Petri, Haiman \& May (2016, 2017), Giocoli et al. (2017, 2018), and Castro et al. (2018), we construct the convergence map weighting the lens planes by the lensing kernel and assuming the Born approximation (Bartelmann \& Schneider 2001). In this respect, as discussed in Giocoli et al. (2016) and Castro et al. (2018) the Born approximation is a very good estimate of the convergence power spectrum and of the probability distribution function (hereafter PDF) down to scales of a few arcseconds. In addition Schäfer et al. (2012) have demonstrated, by computing an analytic perturbative expansion, that the Born approximation is an excellent estimation for weak cosmic lensing down to very small scales $\left(l \geq 10^{4}\right)$.

From $\Sigma_{l}$ we can write down the convergence map $\kappa$ at a given source redshift $z_{\mathrm{s}}$ as

$\kappa=\sum_{l} \frac{\Sigma_{l}}{\Sigma_{\text {crit }, 1, \mathrm{~s}}}$,

where $l$ varies over the different lens planes with the lens redshift $z_{l}$ smaller than $z_{s}$ and $\Sigma_{\text {crit,l,s }}$ represents the critical surface density at the lens plane $z_{l}$ for sources at redshift $z_{\mathrm{s}}$ that can be read as

$\Sigma_{\text {crit }, 1, \mathrm{~s}} \equiv \frac{c^{2}}{4 \pi G} \frac{D_{l}}{D_{s} D_{l s}}$,

where $c$ indicates the speed of light, $G$ the Newton's constant, and $D_{l}, D_{s}$, and $D_{l s}$ are the angular diameter distances between observerlens, observer-source, and source-lens, respectively.

In Fig. 1 we display a schematic representation of haloes in a past light-cone from $z=0$ up to $z=4$. The left-hand panel shows all haloes more massive than $6 \times 10^{13} M_{\odot} h^{-1}$ in comoving coordinates, colour coded depending on their mass. The right-hand panel displays the convergence map for $z_{\mathrm{s}}=4$ with superimposed haloes lying within the field of view. Each constructed convergence map has a square aperture of 5 deg by side and is resolved with $2048 \times 2048$ pixels, which gives a pixel angular resolution of approximately 9 arcsec.

The convergence maps for $z_{\mathrm{s}}=1$ of the same light-cone randomization for the various cosmological models are displayed in Fig. 2. The top left-hand panel refers to the $\Lambda \mathrm{CDM}$ simulation. Within each map we tag the corresponding cosmological model. 

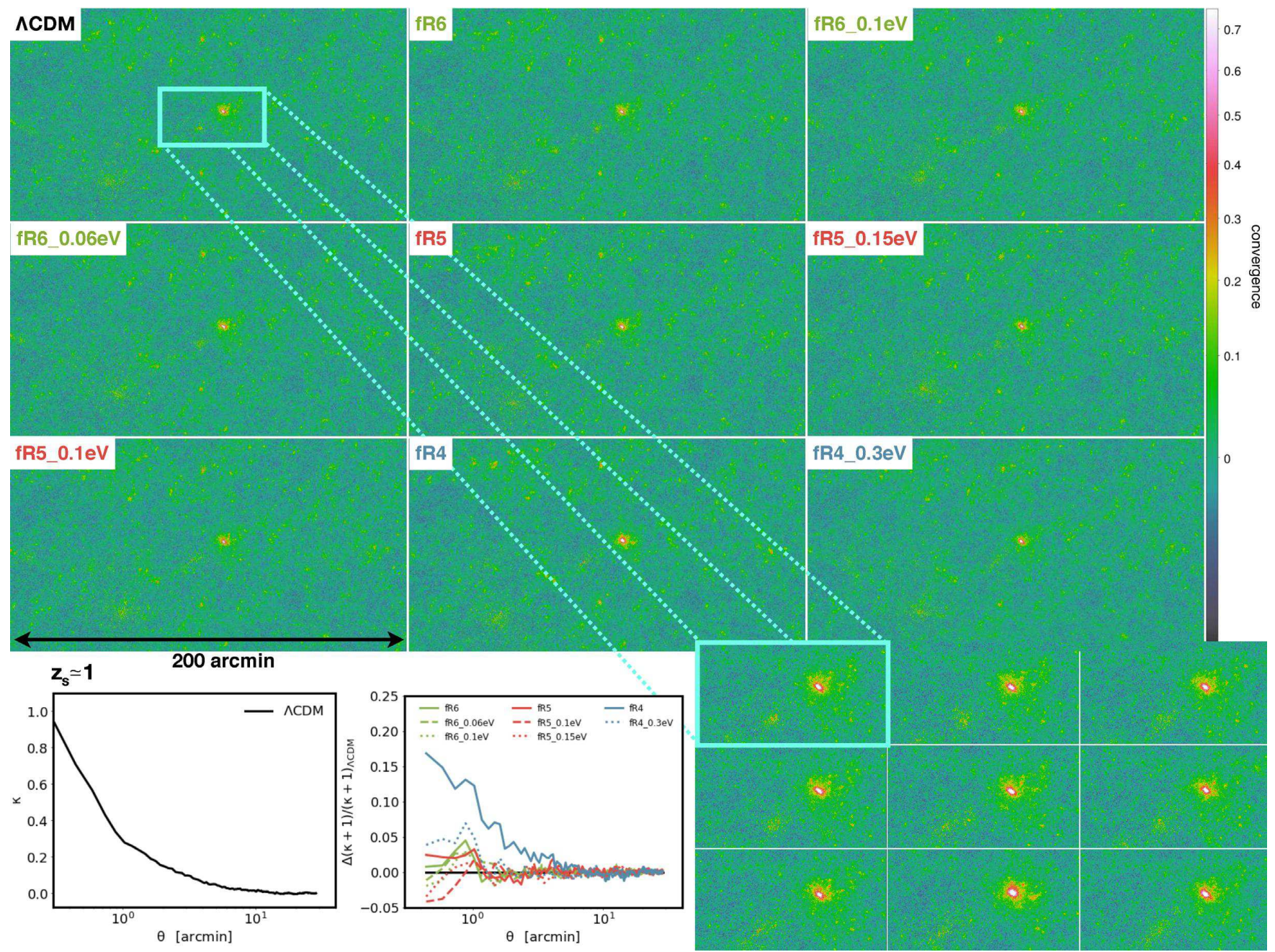

Figure 2. Convergence maps of a light-cone realization for sources at redshift $z_{\mathrm{s}}=1$. The various panels show the convergence maps for the different cosmological simulations, as labelled in the panels. In the subpanel we display a zoom towards an area where a large cluster is located: here small differences between the different models are more visible. The two plots in the bottom left part of the figure show the convergence profile of the cluster in the $\Lambda$ CDM model and the relative difference of $(\kappa+1)$ between the cluster in the various models and the standard reference one. The colour scale refers to the value of the convergence corresponding to each pixel of the maps.

The bottom right-hand subpanel exhibits a zoom towards the centre of the map where we noticed the presence of a massive galaxy cluster with mass $M_{200}=1.14 \times 10^{15} M_{\odot} h^{-1}$ at redshift $z=0.29$. This value refers to the mass enclosing 200 times the critical density of the universe at that redshift in the $\Lambda \mathrm{CDM}$ model; in the other cosmological models the cluster mass has a slightly different value well within 5 per cent except for $f R 5 \_0.15 \mathrm{eV}$ in which it is about 10 per cent smaller and for $f R 4$ in which it is more than 40 per cent larger. The different value of $M_{200}$ in the various models indicates that we are probably observing the same structure in different evolutionary phases: in particular in $f R 5 \_0.15 \mathrm{eV}(f R 4)$ the system is less (more) evolved, which will result in different structural properties like e.g. the concentration parameter (Giocoli et al. 2007). The subpanels on the bottom left part of the figure display the convergence properties of the cluster present at the centre of the maps. The left one shows the convergence $\kappa$ profile in the $\Lambda \mathrm{CDM}$ simulation while the one on the right displays the relative difference between $(\kappa+1)$ profile on the same system in the various models with respect to the $\Lambda \mathrm{CDM}$ measurements. From this figure it is quite evident that the cluster in the $f R 4$ model has a very picked convergence profile that rises 15 per cent more than in the standard cosmology, resulting in a much higher concentration parameter (Barreira et al. 2017).

\subsection{The matter power spectra}

The total matter power spectrum represents a challenging statistics for future wide-field surveys to discriminate between standard and non-standard models. The large amount of data expected from future wide-field surveys, and the great number of available sources for weak lensing measurements expected for the ESA-Mission Euclid (Laureijs et al. 2011), will offer the possibility to constrain with unprecedented accuracy of the dark energy equation of state, the total neutrino mass, and to detect possible deviations from GR by tomographically measuring the growth rate of structures as a function of the weak lensing source redshift.

For each of our cosmological simulations and stored snapshots, we have computed the total matter power spectrum by determining the density field on a cubic Cartesian grid with twice the resolution of the Particle Mesh (hereafter PM) grid used for the $N$-body integration (i.e. $768^{3}$ grid nodes) through a Cloud-in-Cell mass 


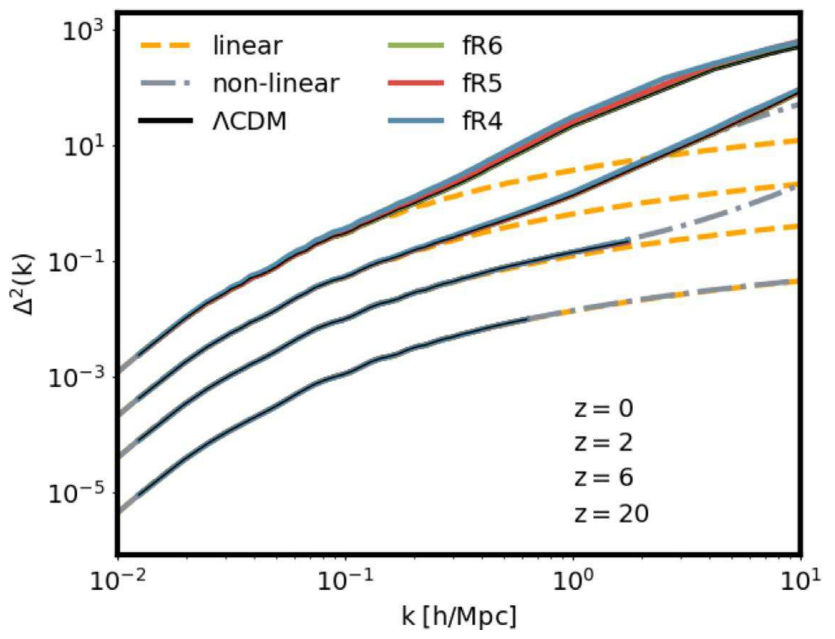

Figure 3. The dimensionless matter power spectra at four different redshifts, $z=0,2,6,20$ from top to bottom. Different colours display the results for the various MG models: $f R 6$ (green), $f R 5$ (red), and $f R 4$ (blue). Black curves display the prediction for the $\Lambda \mathrm{CDM}$ simulation while the orange dashed and dark-grey dot-dashed ones show the linear and non-linear matter power spectrum at the corresponding redshifts from CAMB (Lewis et al. 2000). For the non-linear matter power spectrum we have considered the implementation by Takahashi et al. (2012).

assignment. This procedure provides a determination of the nonlinear matter power spectrum up to the Nyquist frequency of the $\mathrm{PM}$ grid, corresponding to $k_{N y}=\pi N / L \approx 3.2 \mathrm{~h} \mathrm{Mpc}^{-1}$. The obtained power spectrum is then truncated at the $k$-mode where the shot noise reaches 20 per cent of the measured power.

Fig. 3 shows the dimensionless matter power spectra at four different redshifts for the pure MG and the standard $\Lambda$ CDM models. From top to bottom we display redshift $z=0,2,6$, and 20, respectively, which present a constant shift equal to the square of the linear growth factor. The black lines display the measurements for the $\Lambda \mathrm{CDM}$ simulation; green, red, and blue lines refer to the analyses for the MG models $f R 6, f R 5$, and $f R 4$, respectively. The orange dashed and the dark-grey dot-dashed curves show the predictions from linear and non-linear theory at the corresponding redshifts computed using CAMB (Lewis et al. 2000), for the same cosmological parameters of the $\Lambda \mathrm{CDM}$ run. Specifically, for the non-linear matter power spectrum we have adopted the parametrization by Takahashi et al. (2012) which is in agreement with the $\Lambda \mathrm{CDM}$ measurements within few percents (apart for the particle noise contribution appearing above $k \gtrsim 5 h / \mathrm{Mpc}$ for $z=2$ ), as well as the halo model predictions by Mead et al. (2015) not displayed to avoid overcrowding the figure.

The relative differences between the various models and the $\Lambda \mathrm{CDM}$ one are displayed in Fig. 4. In the top left-hand panel we show the differences for the pure MG models, together with the non-linear predictions from MG-CAMB displayed by the corresponding dashed curves. In these panels we notice that MG-CAMB predicts quite well the large-scale power spectra behaviour up to $k$ $\sim 1 \mathrm{Mpc} h^{-1}$ while the small scale trends stay above the simulation measurements. This is a well-known feature related to the onset of the Chameleon screening mechanism at non-linear scales that was already noticed and highlighted in the very first simulations of $f(R)$ gravity (see e.g. Fig. 2 of Oyaizu et al. 2008) and subsequently confirmed by several other studies (see e.g. Zhao, Li \& Koyama 2011). More quantitatively, from the figure we notice that the $f R 4$ model displays at $z=0$ the typical enhancement of $\sim 50$ per cent with respect to the standard model at $k \approx 6 h \mathrm{Mpc}^{-1}$ (Oyaizu et al. 2008), at $z=2$ the relative difference moves towards smaller scales, $k \approx 60 \mathrm{~h} \mathrm{Mpc}^{-1}$, decreasing approximately by a factor of two. The $f R 5$ model for all redshifts $z \neq 0$ remains close to the standard $\Lambda \mathrm{CDM}$, while at the present time it shows an enhancement that monotonically increases from $k=0.1 \mathrm{~h} \mathrm{Mpc}^{-1}$ reaching 25 per cent at $k=10 h \mathrm{Mpc}^{-1}$. The $f R 6$ at all redshifts and scales remains very close to the standard model.

In the other three panels we include also the models that account for the combined effects of MG and massive neutrinos, as indicated in the corresponding labels. In those panels we also display in solid orange some combined predictions of MG-CAMB and non-linear CAMB using the halo model by Mead et al. (2016) for the massive neutrinos runs; specifically, in those cases we are assuming to be able to simply add on top of each other the relative contribution of MG-CAMB to the Mead et al. (2016) predictions. At high redshifts, where the effect of $f(R)$ gravity is still very weak even for the largest values of the scalar amplitude $f_{R 0}$ under consideration, this combined prediction follows quite well the fully non-linear result (see e.g. the $f R 4 \_0.3 \mathrm{eV}$ case down to $z=2$ ). However, at lower redshifts we can observe that the simple sum of the two non-standard predictions fails in capturing the real evolution of the matter power spectrum, in particular at low redshifts, mainly towards large $k$. This demonstrates the need to employ full numerical solutions, or alternatively some faster approximate methods such as those developed by Wright et al. (2017), to investigate and accurately predict non-linear observables in these combined cosmological scenarios. The main qualitative statement arising from the analysis of Fig. 4 is that in all simulations where also massive neutrinos are included the relative difference of the $f(R)$ gravity power spectrum with respect to GR is suppressed. At the same time it is worth to notice that the relative contribution of MG and massive neutrinos to the total matter power spectrum is redshift dependent. In particular, focusing our attention on the simulation $f R 4 \_0.3 e V$, we see that at high redshifts neutrinos tend to lower the power on large scales by about $15-20$ per cent, while at low redshifts the MG contribution acts on small scales producing at the present time a total matter power spectrum very close (within few percent) to the $\Lambda \mathrm{CDM}$ one.

To summarize, in this section we have noticed that $f(R) \mathrm{MG}$ and massive neutrinos affect the matter density fluctuation field at similar scales but with opposite effects and with a slightly different redshift evolution. While MG results in an enhancement of the matter power spectrum at scales smaller than the associated Compton wavelength, massive neutrinos tend to suppress the matter power spectrum on a similar range of scales so that their combined effect may result in a significant weakening of their individual characteristic footprints. These results are all fully consistent with previous findings (see e.g. Baldi et al. 2014; Wright et al. 2017).

Although providing useful insights on the effects of these two phenomena, the comoving 3D matter power spectrum that we have discussed so far is not a directly observable quantity. Present and future wide-field cosmological surveys typically measure projected statistics of the matter density distribution and clustering properties of 3D galaxy catalogues within a past light-cone. Therefore, in order to mimic real observational experiments, we have constructed a set of past light-cones for each cosmological simulation, and in the next sections we will focus on the statistical analysis of weak lensing and cluster counts observables within these light-cones, thereby extending the investigation of the degeneracy from the comoving matter distribution studied in previous works to the more realistic case of weak lensing statistics. 

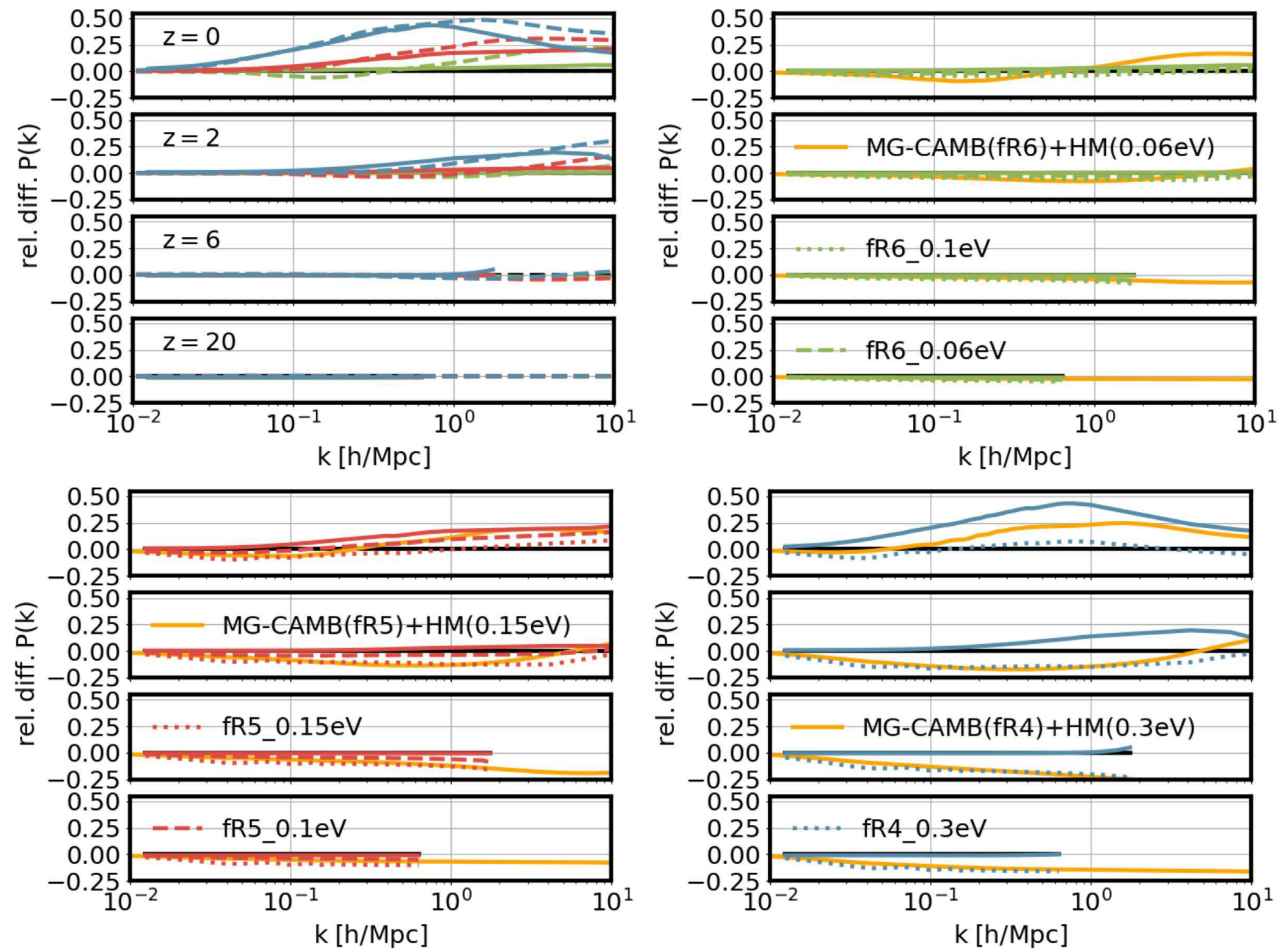

Figure 4. Relative differences between the matter power spectra of a given cosmological model with respect to the $\Lambda \mathrm{CDM}$ one. The panels from top to bottom display the results for $z=0,2,6$, and 20, respectively. The top left-hand panels display the relative differences of the pure MG models, while the others - for each MG case, green, red, and blue for $f R 6$, $f R 5$, and $f R 4$, respectively - show the differences when also a massive neutrinos component is included.

\section{RESULTS}

In this section we present the statistical properties of haloes and projected particle density distribution within constructed past lightcones. These analyses will help us shed more light on "what and where to look at' to better disentangle the various models with respect to the standard $\Lambda \mathrm{CDM}$ reference scenario.

\subsection{Halo mass functions and redshift distribution}

The effect of MG on the dynamical evolution of the matter density field results in different halo formation epochs and number density of collapsed systems. In this respect it is important to mention that cluster counts represent a promising statistics for future surveys, as their number density and redshift distributions are expected to significantly improve the constraints on standard cosmological parameters coming from other probes, as e.g. galaxy clustering and weak lensing (see e.g. Sartoris et al. 2016, for the case of the Euclid survey).

In Fig. 5 we show the median 2D halo mass function per square degree, over 256 realizations, obtained by populating the past lightcones with our halo catalogues up to $z=0.5$. The top panel shows the measurements for the $\Lambda \mathrm{CDM}$ cosmology and the three pure $\mathrm{MG}$ models, in the three bottom subpanels we show the relative differences of the corresponding MG models with and without massive neutrinos with respect to the $\Lambda \mathrm{CDM}$ case. The shaded regions bracketing the black lines mark the first and the third quartiles of the measurements in the $\Lambda \mathrm{CDM}$ simulation computed in the different light-cone randomizations, the red and pink regions display the Poisson uncertainties in halo counts in 25 and $15000 \mathrm{deg}^{2}$, respectively; the latter represents the area of the ESA-Euclid wide survey (Laureijs et al. 2011). The dot-dashed magenta curve shows the theoretical prediction by Despali et al. (2016) for the corresponding mass overdensity definition, which describes quite well the numerical simulation results for the corresponding $\Lambda \mathrm{CDM}$ cosmology down to approximately $10^{13} M_{\odot} h^{-1}$, which corresponds to haloes resolved with at least 125 dark matter particles. From the first subpanel we notice that for masses above $M_{200}>2 \times 10^{13} M_{\odot} h^{-1}$ all $f R 6$ models are quite close, and within the grey region, to the $\Lambda \mathrm{CDM}$ cosmology, sharing a very similar value of $\sigma_{8}$ within few percents; however at smaller masses they tend to predict significantly more haloes, with an increase of about $20-30$ per cent for $M_{200}<10^{13} M_{\odot} h^{-1}$. Differently from this first case, the second subpanel, referring to the $f R 5$ cosmologies, does not show a monotonic trend: while for small and large masses the counts are close to those in the $\Lambda \mathrm{CDM}$ model, for $10^{13} M_{\odot} h^{-1}<M_{200}<10^{14} M_{\odot} h^{-1}$ 

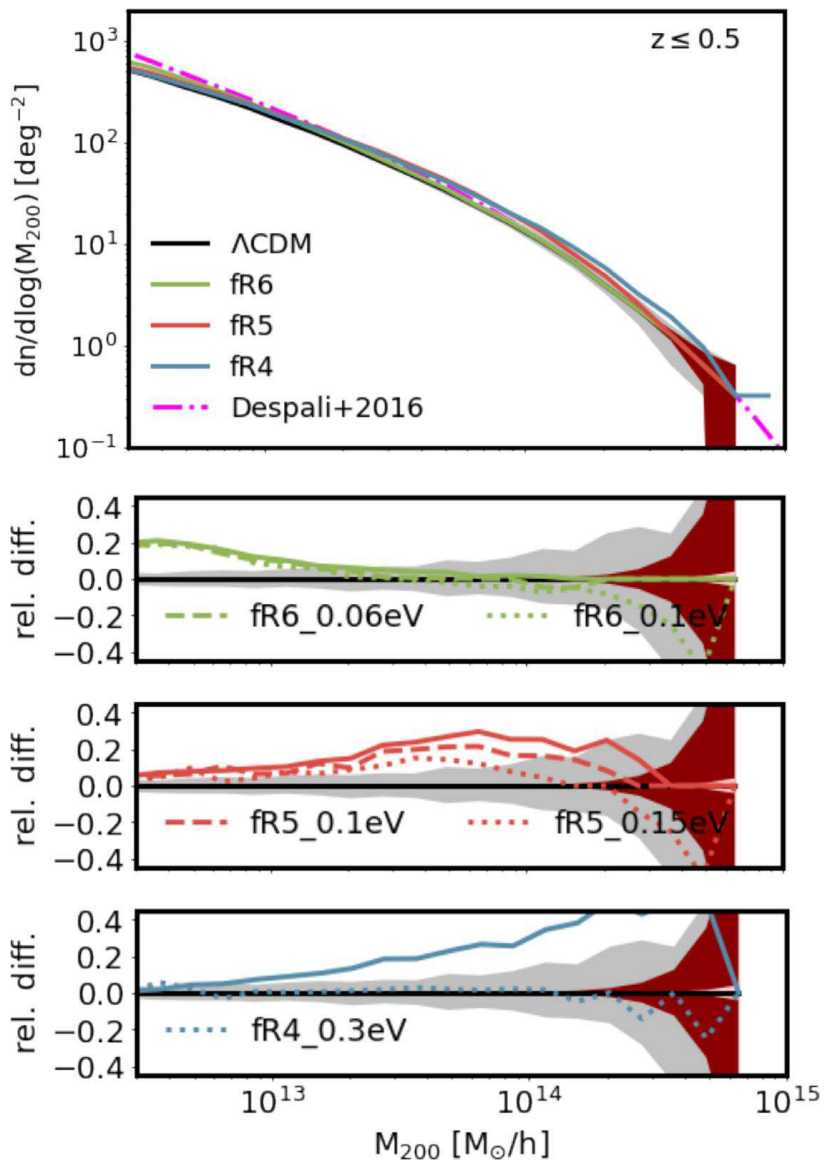

Figure 5. Halo mass function per unit square degree within the past lightcone up to $z=0.5$, in the various cosmological models. The curves display the median counts in the 256 different light-cone realizations, the grey shaded area surrounding the $\Lambda \mathrm{CDM}$ measurements define the first and the third quartiles of the distribution. The red and pink regions mark the Poisson uncertainties of the halo counts within 25 and $15000 \mathrm{deg}^{2}$ (angular size of the Euclid wide survey, Laureijs et al. 2011). The dot-dashed magenta curve shows the prediction for the $\Lambda \mathrm{CDM}$ model computed using the Despali et al. (2016) mass function.

the $f R 5$ models show $10-35$ per cent more haloes with a difference decreasing as a function of the total neutrino mass. In the last subpanel we display the measurements in the $f R 4$ models; while the pure $f R 4$ simulation monotonically shows more haloes than the $\Lambda \mathrm{CDM}$ case, consistently with the larger value of $\sigma_{8}$; the $f R 4 \_0.3 \mathrm{eV}$ case remains very close to the standard cosmology, as expected due to the well-known degeneracy between these two values of $f_{R 0}$ and $m_{v}$ (Baldi et al. 2014; Hagstotz et al. 2018). In general we notice that while MG can have qualitatively different effects for different halo masses depending on the value of the $f_{R 0}$ parameter, the effect of a massive neutrino component is always an increasing suppression of the halo counts for increasing mass, with magnitude that is stronger for larger total neutrino masses.

As discussed in Sartoris et al. (2016), future wide-field surveys are expected to provide important information also on the cluster and halo counts at higher redshifts. For the Euclid wide survey most of the clusters are expected to have a redshift between $0.6<z<$ 1.2 , and even larger.

In order to understand the effect of MG with and without massive neutrinos at these higher redshifts, in Fig. 6 we display the halo mass function per unit square degree for $0.75<z<1.25$. The relative
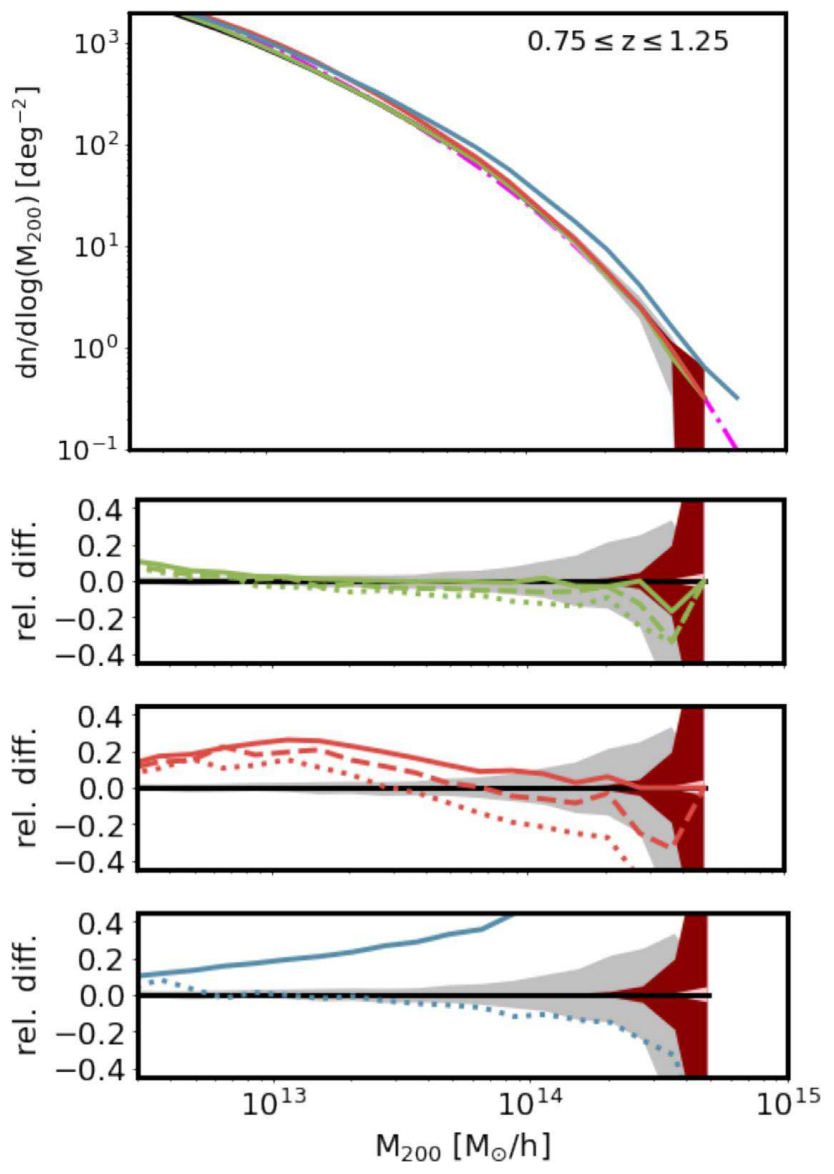

Figure 6. As Fig. 5 but for haloes between $z=0.75$ and $z=1.25$.

trends with respect to $\Lambda \mathrm{CDM}$ and to lower redshift counts allows us to better trace the growth of structures in non-standard models. The data in the panels are analogous to Fig. 5. From Fig. 6 we notice that in particular the $f R 6$ models show a lower excess of low-mass haloes with respect to the standard cosmology as compared to the lower redshift observations of Fig. 5. In this redshift bin the low-mass systems of the $f R 5$ models are more numerous by about 20 per cent while the abundance of more massive cluster-sized objects is again consistent with the $\Lambda \mathrm{CDM}$ expectation. In the last subpanel we show the case of the $f R 4$ cosmologies: the model without massive neutrinos has more haloes than $\Lambda \mathrm{CDM}$, reaching a difference of about 80 per cent for cluster-sized haloes, while the model featuring a $0.3 \mathrm{eV}$ neutrino mass is again very close to the standard cosmology also at these redshifts. These results clearly confirm the strong degeneracy between $f(R)$ MG and massive neutrinos in the nonlinear regime of structure formation that has been first pointed out by Baldi et al. (2014) and subsequently confirmed by other studies (see e.g. Mead et al. 2016; Bellomo et al. 2017; Wright et al. 2017; Peel et al. 2018).

Next generation of space missions, like the Euclid ESA mission, are expected to use cluster counts as a complementary cosmological probe to weak lensing and galaxy clustering. As discussed in Sartoris et al. (2016), photometric identification of galaxy clusters is expected to deliver a catalogue of systems with $S / N>3$ with a minimum mass of approximately $M_{\min } \approx 10^{14} M_{\odot} h^{-1}$, almost independently of redshift up to $z=2$. In Fig. 7 we display the median cluster redshift distribution in the different light-cone realizations. The black curve shows the median counts in the $\Lambda \mathrm{CDM}$ 

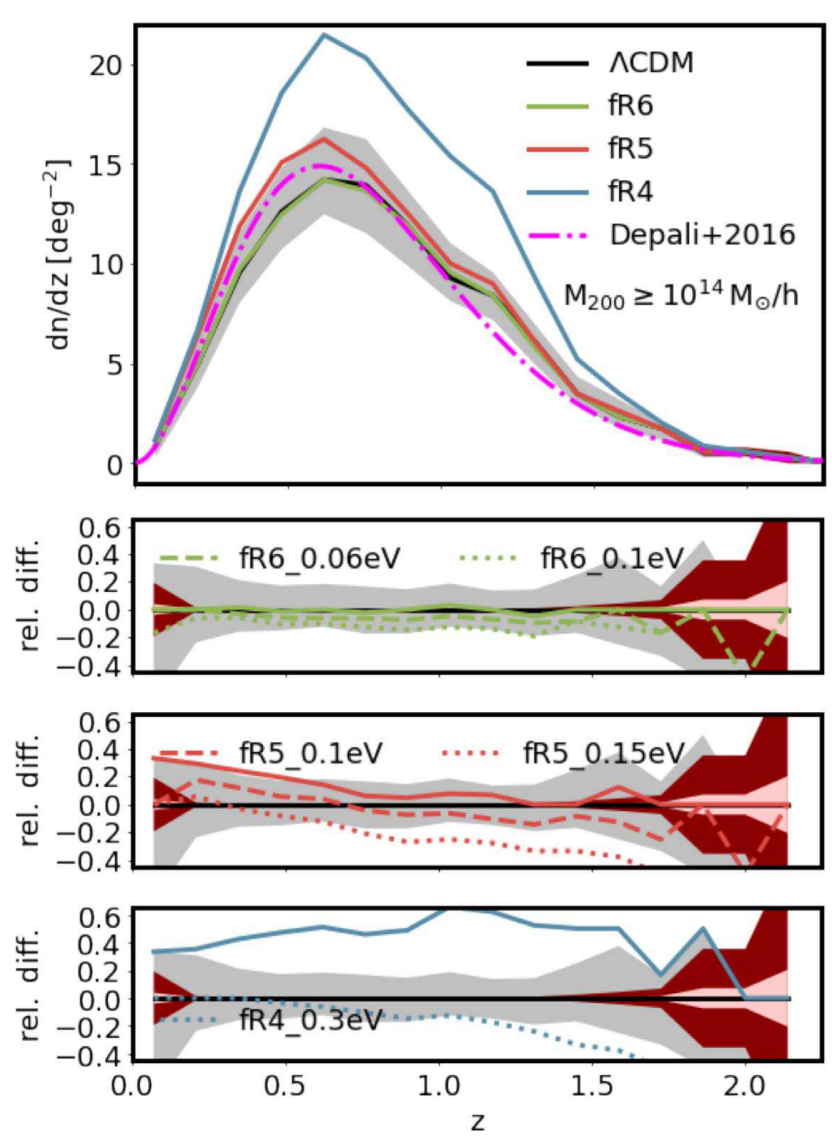

Figure 7. Cluster - haloes more massive than $10^{14} M_{\odot} / h$ - redshift distribution for the various cosmological models. The various curves display the median counts in the different light-cone realizations while the shaded grey area bracketing the $\Lambda \mathrm{CDM}$ measurements defines the first and the third quartiles of the distribution. The red and the pink area mark the Poisson uncertainties of the counts within 25 and $15000 \mathrm{deg}^{2}$. The three subpanels display the relative difference of the counts in the different MG models, with or without the massive neutrino components, with respect to the $\Lambda \mathrm{CDM}$ ones.

past light-cones and the shaded grey area brackets the first and the third quartiles of the distribution. The red and pink regions display the Poisson uncertainties of the cluster counts for a survey of 25 and $15000 \mathrm{deg}^{2}$. Colours and line styles are as in the previous figures, and as before the dot-dashed magenta curve corresponds to the prediction by Despali et al. (2016). The three subpanels show the relative difference of the various cosmological models with respect to the standard one. We may notice that all fR6 and $f R 5$ models show a cluster redshift distribution consistent with $\Lambda \mathrm{CDM}$ well within the sample variance except for the case of $f R 5 \_0.15 \mathrm{eV}$ that presents about 20 per cent fewer clusters towards high redshifts. The $f R 4$ cosmology without massive neutrinos, shows approximately 40 per cent more cluster than $\Lambda \mathrm{CDM}$, while for a neutrino mass of $0.3 \mathrm{eV}$ the cluster redshift distribution appears similar to that of the $f R 5 \_0.15 \mathrm{eV}$ cosmology. This is another example of the observational degeneracy we aim to investigate with the DUSTGRAIN simulations: different values of the total neutrino mass may be inferred from the same data by changing the assumptions on (or even just by adjusting he parameters of) the underlying theory of gravity. A more detailed characterization of the halo mass function in combined $f(R)$ and massive neutrinos cosmologies - calibrated on the same DUSTGRAIN-pathfinder simulations described in this work - has been recently presented in a companion paper by Hagstotz et al. (2018).

\subsection{Properties of the convergence maps}

The shape measurement of a large sample of background sources is known to provide an almost unbiased estimate of the shear field caused by the interposed matter density distribution along the line of sight. In the weak lensing regime, the ellipticity of a galaxy $\varepsilon$ can be written as (Seitz \& Schneider 1997)

$\epsilon \approx \epsilon^{s}+\frac{\gamma}{1-\kappa}$

where $\varepsilon^{s}$ represents the intrinsic ellipticity in the source plane and $\gamma=\left(\gamma_{1}, \gamma_{2}\right)$ is the value of the shear; by defining the lensing potential $\psi$ as the projected potential of the 3D matter density distribution (Kilbinger 2015), we can write the components of $\gamma$ as

$\gamma_{1}=\frac{1}{2}\left(\partial_{1} \partial_{1}-\partial_{2} \partial_{2}\right) \psi, \quad \gamma_{1}=\partial_{1} \partial_{2} \psi$,

as well as for the convergence

$\kappa=\frac{1}{2}\left(\partial_{1} \partial_{1}+\partial_{2} \partial_{2}\right) \psi$,

where $\partial_{i}$ represents the partial derivative with respect to the $i$ th component in the plane of the sky. Averaging on a large sample of galaxies, and considering that the intrinsic ellipticity of the source galaxies has no preferred orientation, we can rewrite equation (18) as

$\langle\epsilon\rangle \approx g$,

where $g \equiv \gamma /(1-\kappa)$ represents the reduced shear. From the measured reduced shear field $g$, suitable algorithms (like e.g. the KSB: Kaiser, Squires \& Broadhurst 1995) are used to recover the convergence map. The weak lensing convergence field is the result of inhomogeneities in the large-scale matter distribution along the line of sight to distant sources.

The convergence power spectrum, to first order, can be expressed as an integral of the $3 \mathrm{D}$ matter power spectrum computed from the observer looking at the past light-cone from the present epoch up to a given source redshift (Bartelmann \& Schneider 2001). In this approximation, it is assumed that the light rays travel along unperturbed paths and all terms higher than first order in convergence and shear can be ignored. Defining $f(w)$ as the angular radial function that depends on the comoving radial coordinate $w$ given the curvature of the universe, we can write the convergence power spectrum for a given source redshift $z_{s}$ - with a corresponding radial coordinate $w_{s}$ - as

$P_{\kappa}(l)=\frac{9 H_{0}^{4} \Omega_{m}^{2}}{4 c^{4}} \int_{0}^{w_{s}\left(z_{s}\right)} \frac{f^{2}\left(w_{s}-w\right)}{f^{2}\left(w_{s}\right) a^{2}(w)} P_{\delta}\left(\frac{l}{f(w)}, w\right) \mathrm{d} w$.

Analogously, from the constructed effective convergence maps we can compute the corresponding power spectrum as

$\left\langle\hat{\kappa}(\mathbf{l}) \hat{\kappa}^{*}\left(\mathbf{l}^{\prime}\right)\right\rangle=4 \pi^{2} \delta_{D}\left(\mathbf{l}-\mathbf{I}^{\prime}\right) P_{\kappa}(l)$,

where $\delta_{D}^{(2)}$ represents a Dirac delta function in 2D.

In Fig. 8 we display the convergence power spectra at four different source redshifts: $z_{\mathrm{s}}=4,2,1$, and 0.5 , from top to bottom, respectively. The black curve represents the average measurements over the 256 different light-cone realizations in the $\Lambda$ CDM model. The black vertical line marks the angular mode corresponding to 


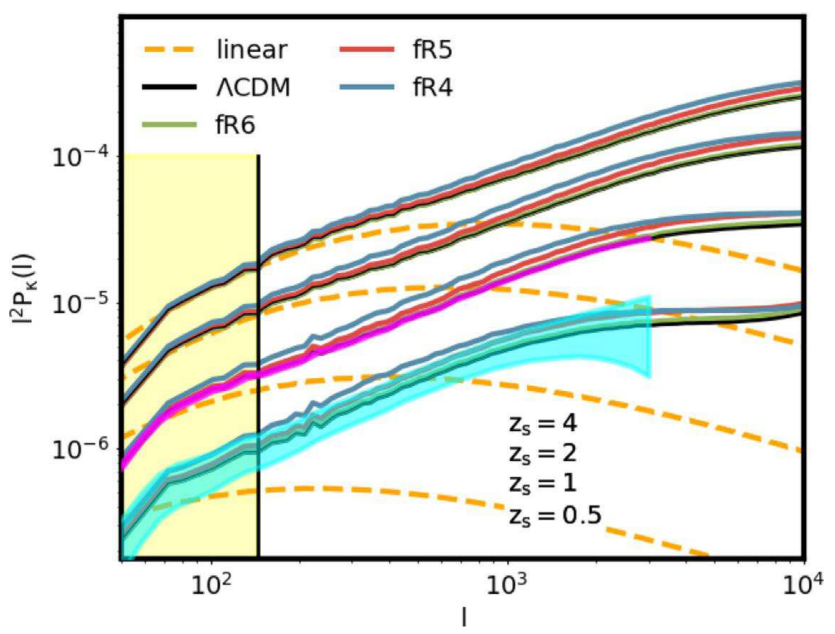

Figure 8. Convergence power spectra at four different redshifts: $z_{\mathrm{s}}=4,2$, 10.5 from top to bottom, respectively. The black curves display the average measurements from 256 light-cone random realizations for the $\Lambda \mathrm{CDM}$ model. Green, red, and blue curves show the measurements for the $f R 6, f R 5$, and $f R 4$ models, respectively; orange dashed curves refer to the prediction using linear matter power spectrum for the $\Lambda \mathrm{CDM}$ cosmology using CAMB. The black vertical line marks the angular mode corresponding to half field of view $l_{\text {half }}=144$. The pink and cyan shaded area illustrate the observational uncertainties - up to $l=3000$ - associated to the power spectra for a survey of 15000 and $154 \mathrm{deg}^{2}$ considering a number density of galaxies of 8 and $33 \mathrm{arcmin}^{-2}$, with corresponding average source redshift of $z_{\mathrm{s}}=1$ and $z_{\mathrm{s}}$ $=0.5$, respectively.

half field of view $l_{\text {half }}=144$. Our light-cone simulations mainly model non-linear scales of the projected matter density field, predictions on larger angular modes can be addressed using linear theory parametrization (Schmidt 2008). The green, red, and blue curves display the average measurements, at the same corresponding redshifts, in the three MG models: $f R 6, f R 5$, and $f R 4$, respectively. The orange dashed curves show the prediction from equation (22) using the theoretical linear power spectrum for the $\Lambda \mathrm{CDM}$ cosmology. The pink and cyan regions, bracketing the $\Lambda \mathrm{CDM}$ predictions at $z_{\mathrm{s}}$ $=0.5$ and $z_{\mathrm{s}}=1$, show the observational uncertainties associated to the shape measurements (Refregier et al. 2004; Lin \& Kilbinger 2015; Zorrilla Matilla et al. 2016; Shan et al. 2018), for a number density of 8 and 33 galaxies arcmin ${ }^{-2}$, mimicking - respectively - a ground-based experiment like CFHTLens (154 deg ${ }^{2}$, Kilbinger et al. 2013) and a future space survey like Euclid (15000 deg ${ }^{2}$, Laureijs et al. 2011). The two wide-field experiments have an average source redshift distribution consistent with the numbers considered above and typically peak around redshift $z=0.5$ and $z=1$, for the ground- and space-based experiments, respectively. Neglecting non-Gaussian corrections we have defined the uncertainties associated with the convergence power spectrum $P_{K}(l)$ (Huterer 2002; Refregier et al. 2004) as

$\Delta P_{\kappa}(l)=\sqrt{\frac{2}{2(l+1) f_{\text {sky }}}}\left(P_{\kappa}(l)+\frac{\sigma_{\epsilon}^{2}}{2 n_{g}}\right)$,

where $f_{\text {sky }}$ represents the fraction of the sky covered by a given survey, $n_{\mathrm{g}}$ the number density of galaxies and $\sigma_{\varepsilon}=0.25$ the rms of the intrinsic ellipticity of the sources.

In Fig. 9 we show the relative differences of the convergence power spectra as computed for the different cosmological models with respect to the standard $\Lambda \mathrm{CDM}$ one. In the top-left panel we display the relative difference of the pure MG models; $z_{\mathrm{s}}$ decreases going from top to bottom. In the subpanels for $z_{\mathrm{s}}=0.5$ and $z_{\mathrm{s}}=$ 1 we display also the shape measurement uncertainties for 8 and 33 galaxies arcmin ${ }^{-2}$, shaded in cyan and pink, respectively, up to $l=3 \times 10^{3}$ that represents the highest angular mode expected to be probed by a survey like Euclid. From this figure we notice that, while for a low-redshift weak lensing survey with a limited source number density it is quite challenging to discriminate between the various MG models, a wide space-based survey with much larger number density of sources for shape measurements will be certainly able to distinguish $f R 4$ and $f R 5$, but still likely not $f R 6$. The other three panels display the relative difference of all the other models with respect to the $\Lambda \mathrm{CDM}$ one, for the same fixed source redshifts. In green, red, and blue we show the $f R 6, f R 5$, and $f R 4$ models with and without massive neutrinos. From the figures we can also notice that it will be very difficult for a future space survey to discriminate the $f R 6$ models with and without massive neutrinos, as well as the $f R 4 \_0.3 \mathrm{eV}$ and the $f R 5 \_0.15 \mathrm{eV}$ (see also our companion paper Peel et al. 2018). This is another example of how the degeneracy between $f(R)$ gravity and massive neutrinos can affect the constraining power of some observables in future cosmological observations, and shows the need of combining primary cosmological probes with other observables like the cluster counts - as discussed in Section 2.2; in addition these observed degeneracies strongly motivate the need of devising more sophisticated statistics of the WL signals that may allow to break them. In this context it will be necessary to look at higher order statistics, probing the shape of the PDF of the convergence that are found to provide a higher discriminating power for the $f(R)-m_{v}$ degeneracy (see e.g. Peel et al. 2018), besides their known feature to break the $\Omega_{\mathrm{M}}-\sigma_{8}$ degeneracy within $\Lambda$ CDM cosmologies (Giocoli et al. 2018; Vicinanza et al. 2018). Alternatively, one may try to exploit a different leverage, namely the possibly different redshift evolution of the $f(R)$ and massive neutrino signals, to disentangle the degenerate models from $\Lambda \mathrm{CDM}$.

From the power spectra at different source redshifts, in fact, we can see that the relative difference with respect to $\Lambda \mathrm{CDM}$ changes, as a consequence of the redshift evolution of $\mathrm{MG}$ and massive neutrinos effects. In the bottom right-hand panel we see that the peak in the relative difference of the projected power spectra of $f R 4$ moves to smaller scales (larger $l$ modes) when the source redshift increases.

The relative difference of the convergence power spectra of the various models at fixed angular mode as a function of the source redshift is displayed in Fig. 10. Left- and right-hand panels show the relative difference of the various models with respect to the $\Lambda \mathrm{CDM}$ one at $l=100$ and $l=1000$, respectively. Almost all models display a monotonic trend, decreasing as a function of the source redshifts. The relative difference of the MG models (without massive neutrinos) tends to be larger at smaller scales - larger $l$, and it is reduced in magnitude by the presence of the massive neutrino components depending on the neutrino mass value. As expected, we notice that the $f R 6$ model presents the smallest difference with respect to the $\Lambda \mathrm{CDM}$ one as a function of $z_{\mathrm{s}}$. The right-hand panel also shows that while for high-redshift sources $f R 6$ is indistinguishable from $\Lambda \mathrm{CDM}$, for $z_{\mathrm{s}}$ moving towards low redshifts the relative difference can be as large as approximately 5 per cent.

In Fig. 11 we display the relative difference of the ratio between the convergence power spectrum at a given source redshift and the power spectrum measured at low redshift, i.e. $z_{\mathrm{s}}=0.5$, for the various cosmological models. Therefore, these plots show the relative growth of the projected matter density distribution with redshift with respect to the standard cosmology. As in the previous 

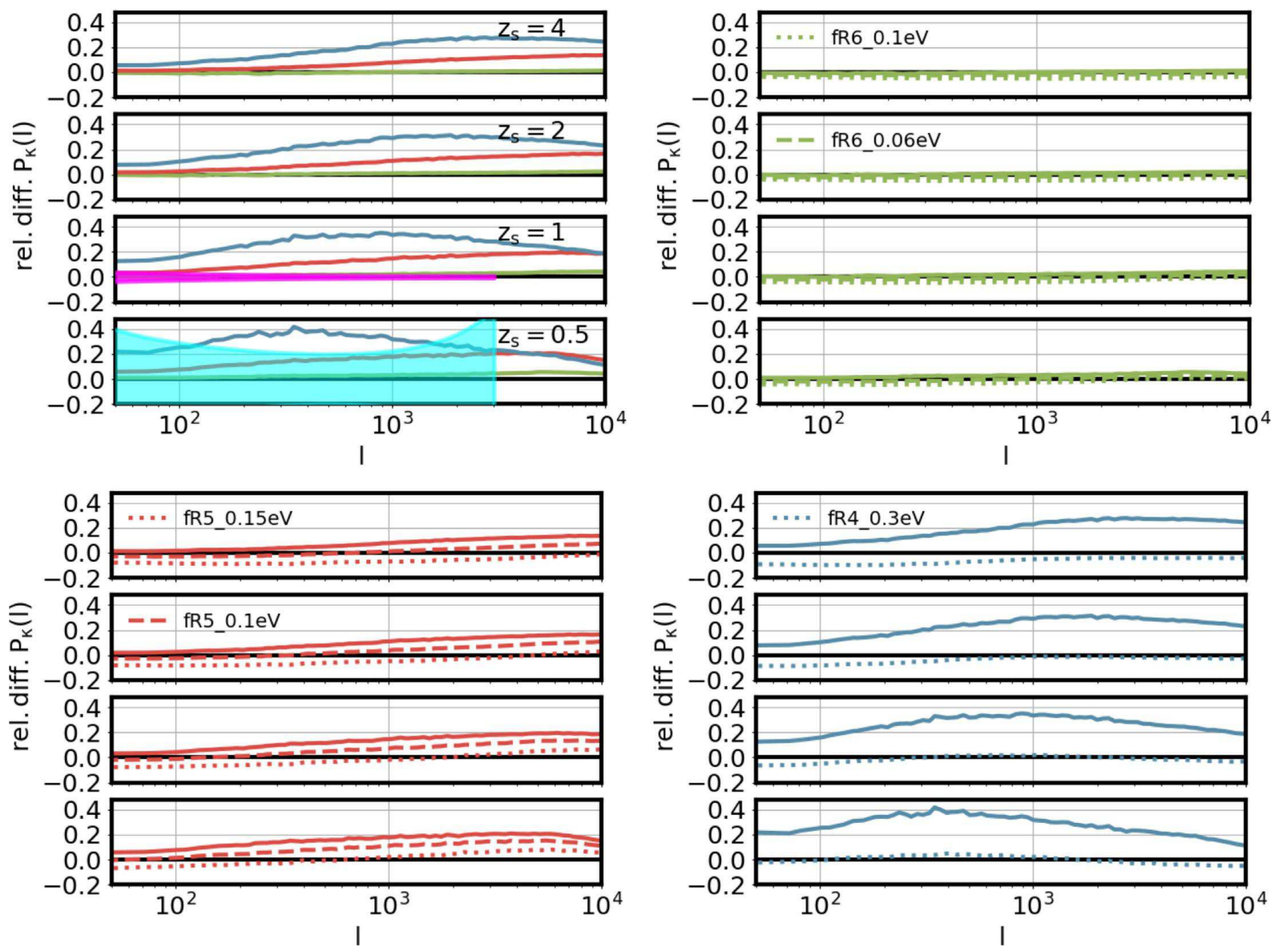

Figure 9. Relative difference between the convergence power spectra at four considered source redshifts for the various cosmological models with respect to the $\Lambda \mathrm{CDM}$ one. In each panel from top to bottom we display the measurements for $z_{\mathrm{s}}=4,2,1$, and 0.5 , respectively. As in Fig. 4 , in the top left-hand panel we display the relative differences for the pure MG models, while in the others we show the MG with the corresponding total massive neutrino components.
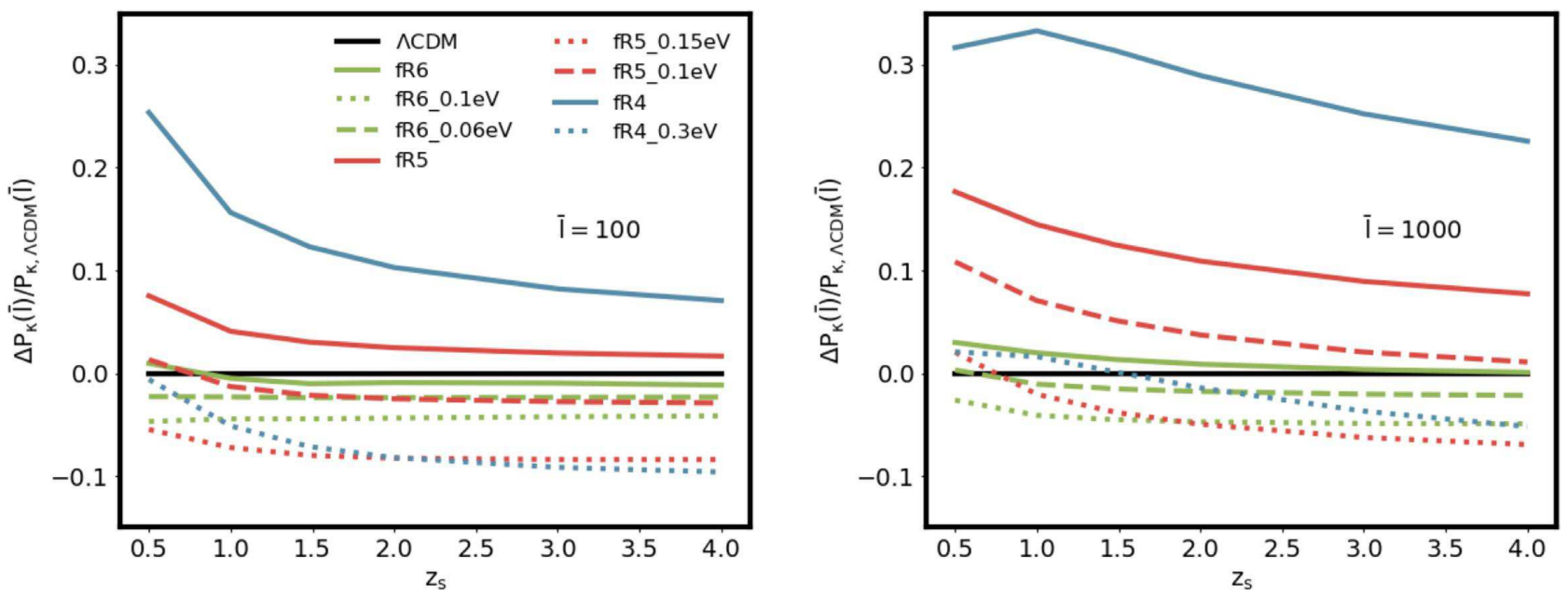

Figure 10. Ratio between the convergence power spectra measured at $l=100$ (left-hand panel) and $l=1000$ (right-hand panel) between the different non-standard models and the $\Lambda \mathrm{CDM}$ one as a function of the source redshift $z_{\mathrm{s}}$.

figure, the top left subpanels show the relative measurements of the pure MG alone, while in the other panels display for each colour the models that account for both MG and massive neutrino components. We can notice that the relative trends are redshift dependent, the difference with respect to $\Lambda \mathrm{CDM}$ is larger at higher source redshifts. Most importantly, we can notice that this kind of tomographic approach allows us to break the degeneracy between $f(R)$ gravity and massive neutrinos, as it can be seen by the fact that the introduction of a massive neutrino component does not make the relative difference with respect to the $\Lambda \mathrm{CDM}$ case to vanish at 

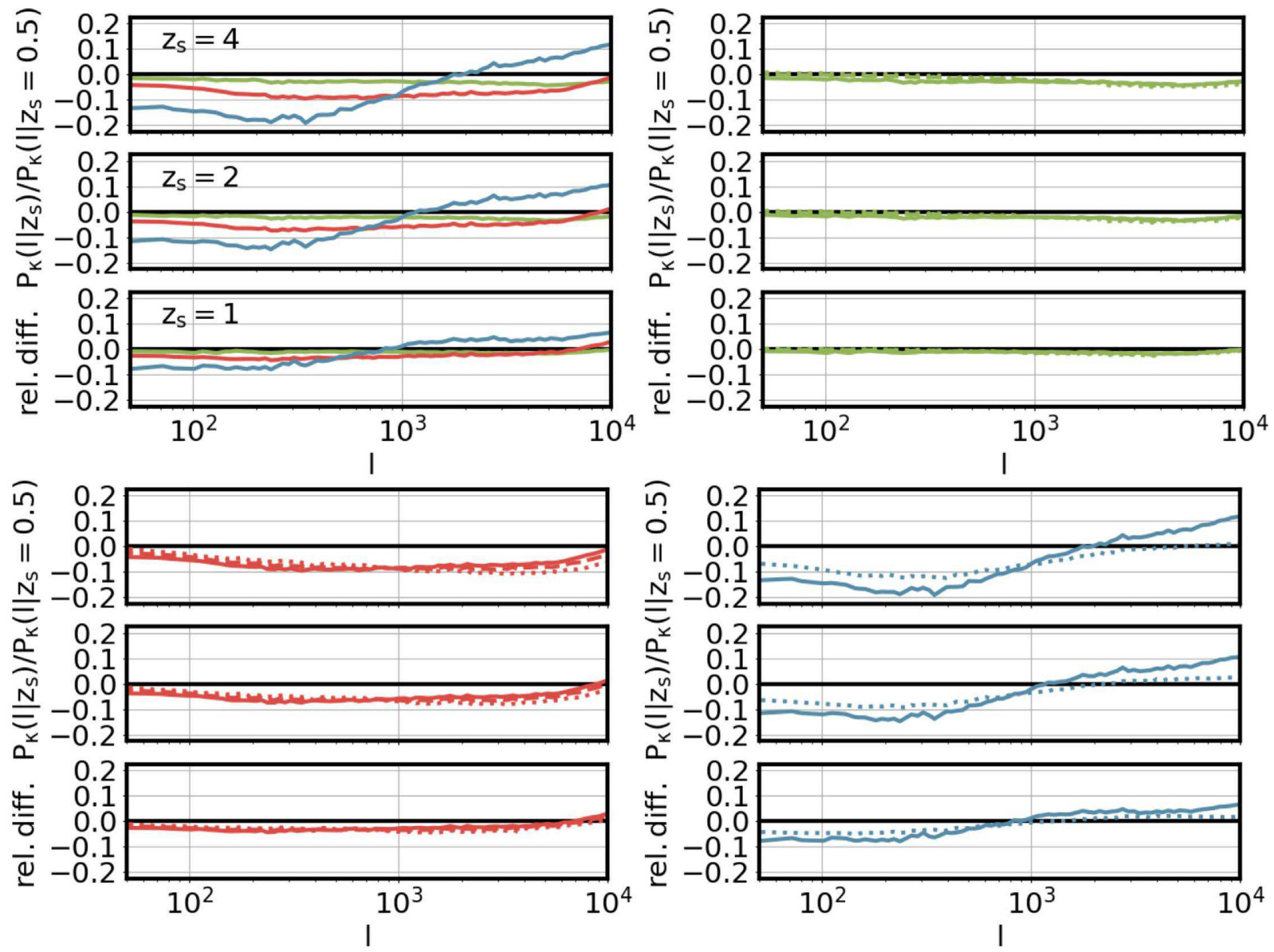

Figure 11. Relative difference between the convergence power spectrum at a given source redshift and that measured considering $z_{\mathrm{s}}=0.5$. Top left-hand panel shows the pure MG models, while the other panels display the cosmologies combining MG and massive neutrinos, colour-coded in green, red, and blue for the $f R 6, f R 5$, and $f R 4$ model, respectively.

all scales, in particular for the $f R 4$ and $f R 5$ models, where a clear signature of the MG effect remains detectable.

In Fig. 12 we show the PDF of the relative difference of the convergence maps constructed at three source redshift $z_{\mathrm{s}}=1,2$, and 4 . To avoid overcrowding the figure we decided not to display the cases for $z_{\mathrm{s}}=0.5$ that do not show particular differences with respect to $\Lambda \mathrm{CDM}$, apart for the extreme MG model $f R 4$. The histograms are computed by binning the quantity $\log (\kappa+1)$ for each pixel between -0.15 and 0.5 in 256 intervals. The panels on the left display the $f R 6$ models, with and without massive neutrinos, from which we can notice that their relative difference with respect to $\Lambda \mathrm{CDM}$ is again very well within the sample variance, represented by the shaded grey regions. The middle panels display the measurements for the $f R 5$ cosmologies, where we can notice a bump for $0.25<\kappa<0.42$, that decreases with the source redshift and with the total neutrino mass. This may be an important signature that can be detectable in the magnification distributions and image parities of strong lensing, as discussed by Castro et al. (2018). In the right-hand panel we display the relative trend of the PDF of the convergence for the $f R 4$ models. The cosmology without massive neutrino component has much more compact non-linear systems and a difference that monotonically increases with the convergence from $\kappa \approx 0.12$ reaching values of 100 per cent already at $\kappa \approx 0.58$. The one-point statistic of the convergence field for the $f R 4 \_0.3 e V$ model shows again a strong degeneracy - within the sample variance - with respect to $\Lambda \mathrm{CDM}$ at low redshifts, while for $z_{\mathrm{s}}=4$ it shows a relative difference reaching $5-10$ per cent, positive for $k \approx 0$ and negative for $k \approx 0.25$. In this final paragraph we have discussed the PDF distributions of the convergence - for various source redshifts; these are simpler to work with and provide complementary information to the convergence power spectra affecting, for instance, the incidence of multiple image events. The cosmological calibration and characterization of the lensing PDFs represent important studies for future wide-fields surveys: they can be used to infer valuable information on the large-scale structure and its evolution through its effect on standard candles (like type Ia Supernovae) since it introduces non-Gaussianities to their scatter (Bernardeau, Van Waerbeke \& Mellier 1997; Hamana \& Futamase 2000; Valageas 2000).

\section{SUMMARY AND CONCLUSIONS}

In this paper we have presented a new suite of cosmological $N$-body simulations - the DUSTGRAIN-pathfinder runs - and we have discussed a first set of results from weak lensing and halo catalogues within past light-cones extracted from their outputs. The DUSTGRAIN project is a numerical enterprise aimed at the investigation of various cosmological observables in the context of cosmological models featuring at the same time a non-standard theory of gravity - here assumed to be in the form of Hu \& Sawicki $f(R)$ - and a non-vanishing neutrino mass, with the final goal of testing observational degeneracies between these two independent phenomena. 

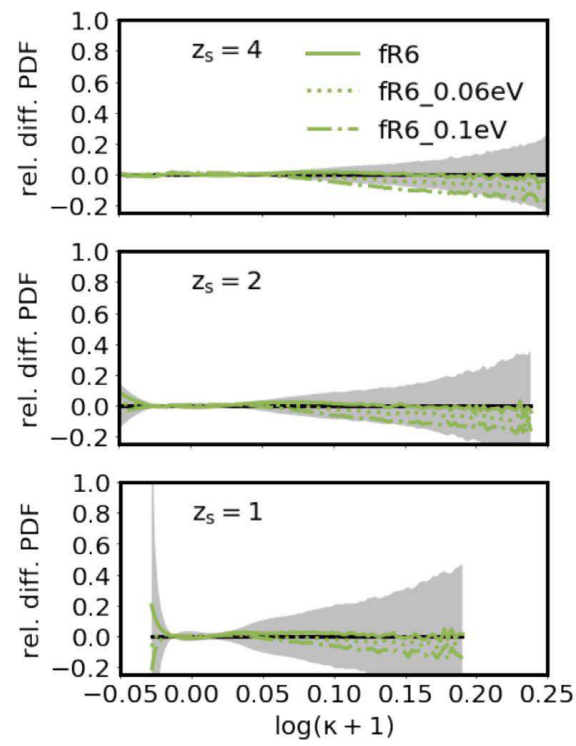
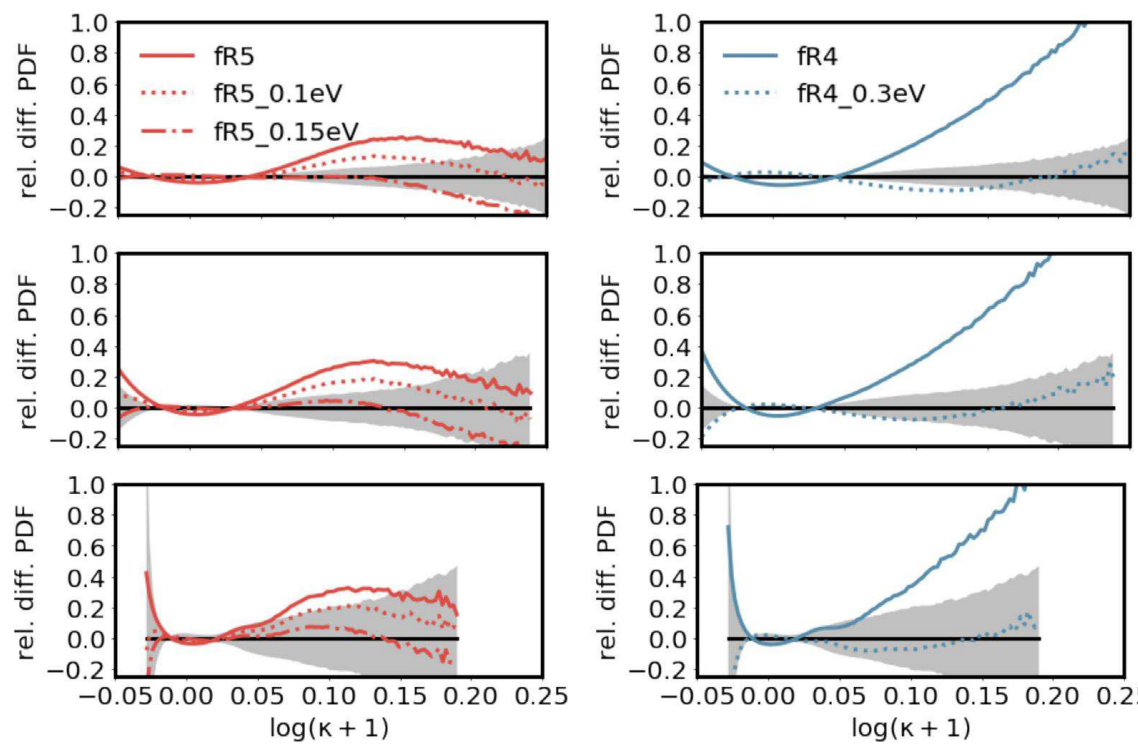

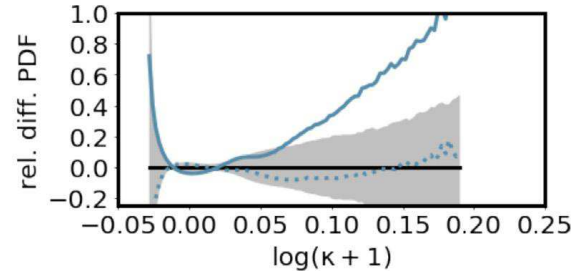

Figure 12. Relative difference of the convergence distribution for the different cosmological models in terms of the $\Lambda$ CDM one. From top to bottom we display the prediction for $z_{\mathrm{s}}=4,2$, and 1 . The curves refer to the average distribution over the various realizations while the shaded area marks the variance of the $\Lambda \mathrm{CDM}$ measurements. The data are constructed binning $\log (\kappa+1)$ between -0.15 and 0.5 in 256 intervals and displayed when for the $\Lambda$ CDM model the counts are larger than 10 units. Green (left-hand panels), blue (central panels), and red (right-hand panels) lines refer to the various MG models, while the corresponding dotted and dashed curves display the results for the combined cosmologies.

In particular, the pathfinder runs are a suite of intermediate-sized simulations aimed at sampling the joint parameter space of these combined models in order to select the most degenerate combinations of their characteristic parameters $f_{R 0}$ and $m_{\mathrm{nu}}$ to be then resimulated with higher dynamical range in the DUSTGRAIN full scale runs. Nonetheless, these preliminary simulations already deliver a wealth of novel information to deserve a detailed analysis.

In particular, in this work we have focused on halo counts and weak lensing statistics within the past light-cones. These extend from the observer - located at $z=0-$ up to redshift $z=4$ with an angular aperture of $5 \times 5 \mathrm{deg}^{2}$ resolved with $2048 \times 2048$ pixels. From each simulation we generated a sample of 256 different realizations randomizing the boxes of the various simulation snapshots.

In what follows we summarize our main results:

(i) the $3 \mathrm{D}$ comoving power spectra show clear signatures of $\mathrm{MG}$ with a difference that increases towards larger scales and lower redshifts; the presence of massive neutrinos, however, slows down the growth rate of perturbation reducing the matter power spectrum mainly at large scales; strongly suppresses these observational footprints; therefore, the matter power spectra show a strong degeneracy with respect to the standard $\Lambda \mathrm{CDM}$ scenario for suitable combinations of MG and massive neutrinos parameters, as already found by previous studies;

(ii) the $2 \mathrm{D}$ projected halo mass function shows different characteristic signatures for different MG models. In particular, the fR6 model shows an increase of the low-mass halo counts while the $f R 5$ model presents at low (high) redshifts an excess of $\approx 10^{14} M_{\odot} h^{-1}$ $\left(\approx 10^{13} M_{\odot} h^{-1}\right)$ haloes and the $f R 4$ run displays a monotonic increase of the halo mass function in the light-cone with respect to the standard $\Lambda C D M$ with a clear excess of high-mass haloes; the inclusion of massive neutrinos always suppresses the counts of high-mass haloes resulting in a closer agreement with respect to the reference $\Lambda \mathrm{CDM}$ model, except for $f R 6$;

(iii) the redshift distribution of galaxy clusters (haloes with $M_{200}$ $\left.>10^{14} M_{\odot} h^{-1}\right)$ in the $f R 4$ model is very different from $\Lambda \mathrm{CDM}$ with an excess of approximately 40 per cent with respect to the reference model; all the other cosmologies present a redshift distribution of galaxy clusters within the sample variance of the $\Lambda \mathrm{CDM}$ one apart from $f R 5$ with $m_{v}=0.15 \mathrm{eV}$ and $f R 4$ with $m_{v}=0.3 \mathrm{eV}$ that display a decline of counts towards high redshifts;

(iv) the convergence power spectra show distinct signatures of MG, mainly for high source redshifts, where the different nonlinear properties of the structures are distinguishable for angular modes $l \geq 10^{3}$; also in this case, the presence of massive neutrinos suppresses these signatures, making the measurements closer to the standard reference model;

(v) a tomographic analysis of the power spectra at different source redshifts is found to be very promising in distinguishing some of these degenerate non-standard models from $\Lambda$ CDM by exploiting the different redshift evolution of the $f(R)$ gravity and massive neutrinos effects on large-scale structures formation and evolution; the same is also true for the one-point statistic of the convergence field for different source redshifts.

The analysis of simulated past light-cones of non-standard cosmologies represents a realistic way to assess the capabilities of ongoing and future surveys to disentangle such cosmological models from standard $\Lambda \mathrm{CDM}$. In this work we have analysed cluster counts and weak lensing statistics of $f(R)$ gravity cosmological $N$-body simulations with and without massive neutrinos and compared the findings with a standard reference $\Lambda$ CDM cosmology. In both cluster counts and weak lensing statistics some of the pure $f(R)$ models appear very distinct from $\Lambda \mathrm{CDM}$, but the combination with massive neutrinos can make them to appear again consistent with the standard scenario. These results underline the difficulty of the 'observables' we have looked at to disentangle by themselves these degenerate non-standard models suggesting the need of combining various cosmological probes, or of looking at higher order statistics or cross-correlating different observables, or finally to exploit observations ad different redshifts to break the degeneracies. 


\section{ACKNOWLEDGEMENTS}

CG and MB acknowledge support from the Italian Ministry for Education, University and Research (MIUR) through the SIR individual grant SIMCODE (project number RBSI14P4IH). CG acknowledges support from the Italian Ministry of Foreign Affairs and International Cooperation, Directorate General for Country Promotion. We also acknowledge the support from the grant MIUR PRIN 2015 'Cosmology and Fundamental Physics: illuminating the Dark Universe with Euclid'; and the financial contribution from the agreement ASI n.I/023/12/0 'Attività relative alla fase B2/C per la missione Euclid'. The DUSTGRAIN-pathfinder simulations discussed in this work have been performed and analysed on the Marconi supercomputing machine at Cineca thanks to the PRACE project SIMCODE1 (grant nr. 2016153604) and on the computing facilities of the Computational Center for Particle and Astrophysics (C2PAP) and of the Leibniz Supercomputer Center (LRZ) under the project ID pr94ji. We thank the anonymous reviewer for her/his useful comments.

\section{REFERENCES}

Arnold C., Puchwein E., Springel V., 2014, MNRAS, 440, 833

Arnold C., Puchwein E., Springel V., 2015, MNRAS, 448, 2275

Arnold C., Springel V., Puchwein E., 2016, MNRAS, 462, 1530

Arnold C., Fosalba P., Springel V., Puchwein E., Blot L., 2018, preprint (arXiv:1805.09824)

Baldi M., Villaescusa-Navarro F., 2018, MNRAS, 473, 3226

Baldi M., Villaescusa-Navarro F., Viel M., Puchwein E., Springel V., Moscardini L., 2014, MNRAS, 440, 75

Barreira A., Llinares C., Bose S., Li B., 2016, J. Cosmol. Astropart. Phys., 5,001

Barreira A., Bose S., Li B., Llinares C., 2017, J. Cosmol. Astropart. Phys., 2,031

Bartelmann M., 2010, Class. Quantum Gravity, 27, 233001

Bartelmann M., Schneider P., 2001, Phys. Rep., 340, 291

Bellomo N., Bellini E., Hu B., Jimenez R., Pena-Garay C., Verde L., 2017, J. Cosmol. Astropart. Phys., 2, 043

Benjamin J. et al., 2013, MNRAS, 431, 1547

Bernardeau F., Van Waerbeke L., Mellier Y., 1997, Astron. Astrophys., 322, 1

Bertotti B., Iess L., Tortora P., 2003, Nature, 425, 374

Bertschinger E., Zukin P., 2008, Phys. Rev. D, 78, 024015

Brax P., Valageas P., 2014, Phys. Rev. D, 90, 023508

Broadhurst T. et al., 2005b, ApJ, 621, 53

Buchdahl H. A., 1970, MNRAS, 150, 1

Castro T., Quartin M., Giocoli C., Borgani S., Dolag K., 2018, MNRAS, 478,1305

Cleveland B. T., Daily T., Davis R., Jr., Distel J. R., Lande K., Lee C. K., Wildenhain P. S., Ullman J., 1998, ApJ, 496, 505

Coe D. et al., 2013, ApJ, 762, 32

Costanzi M., Sartoris B., Viel M., Borgani S., 2014, J. Cosmol. Astropart. Phys., 10, 081

Davis M., Efstathiou G., Frenk C. S., White S. D. M., 1985, ApJ, 292, 371

Deffayet C., Dvali G., Gabadadze G., Vainshtein A. I., 2002, Phys. Rev., D65, 044026

Despali G., Giocoli C., Angulo R. E., Tormen G., Sheth R. K., Baso G., Moscardini L., 2016, MNRAS, 456, 2486

Einstein A., 1918, Sitzungsberichte der Königlich Preußischen Akademie der Wissenschaften (Berlin). Seite 448

Erben T. et al., 2013, MNRAS, 433, 2545

Fu L. et al., 2008, A\&A, 479, 9

Giocoli C., Moreno J., Sheth R. K., Tormen G., 2007, MNRAS, 376, 977

Giocoli C., Meneghetti M., Metcalf R. B., Ettori S., Moscardini L., 2014, MNRAS, 440, 1899

Giocoli C. et al., 2016, MNRAS, 461, 209
Giocoli C. et al., 2017, MNRAS, 470, 3574

Giocoli C., Moscardini L., Baldi M., Meneghetti M., Metcalf R. B., 2018, MNRAS, 478, 5436

Hagstotz S., Costanzi M., Baldi M., Weller J., 2018, preprint (arXiv:1806.07400)

Hamana T., Futamase T., 2000, ApJ, 534, 29

Harnois-Déraps J., Munshi D., Valageas P., van Waerbeke L., Brax P., Coles P., Rizzo L., 2015, MNRAS, 454, 2722

He J.-H., 2013, Phys. Rev. D, 88, 103523

Heymans C. et al., 2013, MNRAS, 432, 2433

Higuchi Y., Shirasaki M., 2016, MNRAS, 459, 2762

Hildebrandt H. et al., 2017, MNRAS, 465, 1454

Hinterbichler K., Khoury J., 2010, Phys. Rev. Lett., 104, 231301

Hojjati A., Pogosian L., Zhao G.-B., 2011, J. Cosmol. Astropart. Phys., 8, 005

Hu W., Sawicki I., 2007, Phys. Rev. D., 76, 064004

Huterer D., 2002, Phys. Rev. D, 65, 063001

Kaiser N., Squires G., Broadhurst T., 1995, ApJ, 449, 460

Khoury J., Weltman A., 2004, Phys. Rev. D, 69, 044026

Kilbinger M., 2015, Rep. Prog. Phys., 78, 086901

Kilbinger M. et al., 2013, MNRAS, 430, 2200

Kitching T. D. et al., 2014, MNRAS, 442, 1326

Kitching T. D., Taylor A. N., Cropper M., Hoekstra H., Hood R. K. E., Massey R., Niemi S., 2016, MNRAS, 455, 3319

Landau L. D., Lifshitz E. M., 1971, The Classical Theory of Fields, Pergamon Press, New York

Laureijs R., et al., 2011, preprint (arXiv:1110.3193)

Lesgourgues J., Pastor S., 2006, Phys. Rep., 429, 307

Lewis A., Challinor A., Lasenby A., 2000, ApJ, 538, 473

Li B., Shirasaki M., 2018, MNRAS, 474, 3599

Li B., Zhao G.-B., Teyssier R., Koyama K., 2012, J. Cosmol. Astropart. Phys., 1201, 051

Lin C. A., Kilbinger M., 2015, A\&A, 576, A24

Llinares C., Mota D. F., Winther H. A., 2014, A\&A, 562, A78

Mead A. J., Peacock J. A., Heymans C., Joudaki S., Heavens A. F., 2015, MNRAS, 454, 1958

Mead A. J., Heymans C., Lombriser L., Peacock J. A., Steele O. I., Winther H. A., 2016, MNRAS, 459, 1468

Meneghetti M., Bartelmann M., Dahle H., Limousin M., 2013, Space Sci. Rev., 177, 31

Motohashi H., Starobinsky A. A., Yokoyama J., 2013, Phys. Rev. Lett., 110, 121302

Naik A. P., Puchwein E., Davis A. C., Arnold C., 2018, MNRAS, 480, 5211

Novikov E. A., 2016, Electron. J. Theor. Phys., 13, 79

Oyaizu H., Lima M., Hu W., 2008, Phys. Rev. D, 78, 123524

Peel A., Pettorino V., Giocoli C., Starck J.-L., Baldi M., 2018, preprint (arXiv:1805.05146)

Petri A., Haiman Z., May M., 2016, Phys. Rev. D, 93, 063524

Petri A., Haiman Z., May M., 2017, Phys. Rev. D, 95, 123503

Planck Collaboration I, 2011, A\&A, 536, A1

Planck Collaboration XI, 2013, A\&A, 557, A52

Planck Collaboration I 2014, A\&A, 571, A1

Planck Collaboration XX, 2014, A\&A, 571, A20

Planck Collaboration I, 2016, A\&A, 594, A1

Planck Collaboration XXIV 2016a, A\&A, 594, A24

Planck Collaboration XIII, 2016b, A\&A, 594, A13

Poulin V., Boddy K. K., Bird S., Kamionkowski M., 2018, Phys. Rev. D, 97, 123504

Puchwein E., Baldi M., Springel V., 2013, MNRAS, 436, 348

Refregier A. et al., 2004, AJ, 127, 3102

Roncarelli M., Moscardini L., Borgani S., Dolag K., 2007, MNRAS, 378, 1259

Roncarelli M., Baldi M., Villaescusa-Navarro F., 2018, MNRAS, preprint (arXiv:1805.11607)

Sartoris B. et al., 2016, MNRAS, 459, 1764

Schäfer B. M., Heisenberg L., Kalovidouris A. F., Bacon D. J., 2012, MNRAS, 420, 455

Schmidt F., 2008, Phys. Rev. D, 78, 043002 
Schmidt F., Lima M. V., Oyaizu H., Hu W., 2009, Phys. Rev., D79, 083518 Seitz C., Schneider P., 1997, A\&A, 318, 687

Shan H. et al., 2018, MNRAS, 474, 1116

Shirasaki M., Nishimichi T., Li B., Higuchi Y., 2017, MNRAS, 466, 2402

Sotiriou T. P., Faraoni V., 2010, Rev. Mod. Phys., 82, 451

Springel V., 2005, MNRAS, 364, 1105

Springel V., White S. D. M., Tormen G., Kauffmann G., 2001, MNRAS, 328,726

Starobinsky A. A., 1980, Phys. Lett., B91, 99

Takahashi R., Sato M., Nishimichi T., Taruya A., Oguri M., 2012, ApJ, 761, 152

Valageas P., 2000, A\&A, 356, 771

Vicinanza M., Cardone V. F., Maoli R., Scaramella R., Er X., 2018, Phys. Rev. D, 97, 023519

Viel M., Haehnelt M. G., Springel V., 2010, J. Cosmol. Astropart. Phys., 1006,015

Villaescusa-Navarro F., Banerjee A., Dalal N., Castorina E., Scoccimarro R., Angulo R., Spergel D. N., 2018, ApJ, 861, 53
Wagner C., Verde L., Jimenez R., 2012, ApJ, 752, L31

Will C. M., 2005, Living Rev. Rel., 9, 3

Winther H. A. et al., 2015, MNRAS, 454, 4208

Wright B. S., Winther H. A., Koyama K., 2017, J. Cosmol. Astropart. Phys., 10,054

Zennaro M., Bel J., Villaescusa-Navarro F., Carbone C., Sefusatti E., Guzzo L., 2017, MNRAS, 466, 3244

Zhao G.-B., Li B., Koyama K., 2011, Phys. Rev., D83, 044007

Zhao G.-B., Pogosian L., Silvestri A., Zylberberg J., 2009, Phys. Rev. D, 79,083513

Zorrilla Matilla J. M., Haiman Z., Hsu D., Gupta A., Petri A., 2016, Phys. Rev. D, 94, 083506

This paper has been typeset from a $\mathrm{T}_{\mathrm{E}} \mathrm{X} / \mathrm{L} \mathrm{T} \mathrm{E} X$ file prepared by the author. 